\title{
Framing-Effekte auf dem Prüfstand
}

\author{
Eine theoretische, methodische und empirische Auseinandersetzung \\ mit der Wirkungsperspektive des Framing-Ansatzes
}

\section{Bertram Scheufele}

Der Beitrag fokussiert die Wirkungsperspektive des Framing-Ansatzes. Zunächst werden verschiedene Stränge der Framing-Forschung klassifiziert. Daneben wird eine Definition von „Frame“ und „Framing " geliefert, die psychologische und soziologische Ansätze integriert, operationalisierbar ist und pragmatisch zwischen den Konstrukten „Frame “ und „Schema“ unterscheidet. Mit diesem Rüstzeng lassen sich bisherige Arbeiten zu FramingEffekten theoretisch, methodisch und empirisch diskutieren und erweitern. Die Auseinandersetzung mit der Wirkungsperspektive des Framing-Ansatzes zeigt, dass aktuellere psychologische Konstrukte und Theorien zu wenig aufgegriffen werden, dass der Fokus auf Schema-Aktivierung wichtige Typen von Framing-Effekten übersieht, und dass daher diverse empirische Fragen unbeantwortet bleiben und methodische Möglichkeiten vergeben werden.

Keywords: Frame, Framing, Schema, Medienwirkung, Agenda-Setting, Priming

\section{Perspektiven des Framing-Ansatzes}

Bisherige Systematisierungen der Framing-Forschung (u. a. Entman, 1991, 1993; Scheufele, 2000; D. Scheufele, 1999) sind variablenorientiert, fokussieren also auf (un)abhängige Variablen, ohne hinreichend die Mechanismen von Framing-Effekten oder die disparaten Terminologien und Theoriegrundlagen der Framing-Forschung zu berücksichtigen. Brauchbarer in dieser Hinsicht scheint eine modellgebundene Systematisierung (Scheufele, 2003: 61ff.). ${ }^{1}$

Definitionen von „Frame“ und „Framing“ (z. B. Entman 1993: 52; Gitlin 1980: 7f.; Gamson \& Modigliani, 1989: 3; Pan \& Kosicki 1993: 56f.; Tankard 2001: 110f.; Reese, 2001: 11) lassen sich auf folgenden vorläufigen Nenner bringen: Frames gelten als Interpretationsmuster, mit denen sich Informationen sinnvoll einordnen und effizient verarbeiten lassen. Durch Framing werden bestimmte ,aspects of perceived reality“ (Entman, 1993: 52) betont, während andere ignoriert werden (Snow \& Benford, 1992: 137; Cappella \& Jamieson, 1997: 45f.; Pan \& Kosicki, 1993: 57; Reese, 2001: 11). Dadurch werden Attributionen, Bewertungen oder Entscheidungen nahe gelegt (vgl. Entman, 1993; Iyengar, 1991; Price et al., 1997; Shah et al., 1996; Gamson \& Modigliani, 1989).

Damit kann man Frames bzw. Framing horizontal in drei Bereichen verorten (Scheufele, 1999a: 92f.; Entman, 1993: 52f.; Tuchman, 1978: 193): (1) bei Journalisten bzw. im Mediensystem, (2) bei Rezipienten bzw. der Bevölkerung sowie (3) bei Akteuren, Gruppen und Organisationen in Politik, Wirtschaft, Kultur usw. (Abbildung 1). Vertikal siedeln viele Autoren (z. B. Entman, 1991: 7, 1993: 52f.; Scheufele, 2000; van Dijk, 1988) Frames auf kognitiver und textueller Ebene an. Andere Autoren (z. B. Kinder \& Sanders, 1990: 74; Gamson, 1992) verstehen Frames als Muster öffentlicher Dis-

1 Sie ist vergleichbar mit Synopsen von Rogers \& Dearing (1988) und Manheim (1986) für Agenda-Setting. 
Abbildung 1: Forschungsstränge der Framing-Forschung (Scheufele, 2003)

\begin{tabular}{l|l|l|l}
\hline & \multicolumn{2}{|c}{ (System-)Bereiche (Auswahl) } \\
\hline Ebenen & $\begin{array}{c}\text { Politiker / } \\
\text { Politisches System }\end{array}$ & $\begin{array}{c}\text { Journalisten / } \\
\text { Mediensystem }\end{array}$ & $\begin{array}{c}\text { Rezipienten / } \\
\text { Bevölkerung }\end{array}$ \\
\hline $\begin{array}{l}\text { Kognitive } \\
\text { Ebene }\end{array}$ & 1 & 4 & 7 \\
\hline $\begin{array}{l}\text { Diskursive } \\
\text { Ebene }\end{array}$ & 2 & 5 & \\
\hline $\begin{array}{l}\text { Diskurs- } \\
\text { Produkt }\end{array}$ & 3 & 6 & \\
\hline
\end{tabular}

Hinweis: Schattierungen geben an, welche Ebenen/Bereiche ein Forschungsstrang tangiert.

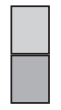

Journalistenzentrierter Ansatz

Inhaltszentrierter Ansatz

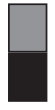

Öffentlichkeits-/

bewegungstheoretischer Ansatz

Wirkungszentrierter Ansatz

kurse. Damit kann man einen Frame vertikal dreifach verorten: (1) als kognitiven „Erwartungsrahmen, der sich aus einzelnen Schemata [...] konstituiert" (Scheufele, 2003: 65), (2) der sich in Kommunikation mit anderen Menschen etabliert und verändert und (3) der sich als „Struktur von Diskursprodukten“ manifestiert (Abbildung 1). ${ }^{2}$ Mit diesen Einordnungen lassen sich drei Hauptstränge der Framing-Forschung benennen (Scheufele, 2003: 65ff.):

- Zur Kommunikatorperspektive gehören zwei Ansätze: (1) Die journalistenzentrierte Perspektive (Scheufele, 2003; Brosius \& Eps, 1993; Gitlin, 1980; Tuchman, 1972, 1978) fragt, wie journalistische Frames (Felder 4 und 5, Abbildung 1) die Nachrichtenproduktion beeinflussen und sich als Medien-Frames (Feld 6) in der Berichterstattung niederschlagen. (2) Die inhaltszentrierte Perspektive (z. B. Entman, 1991; Woo, 1996; Pan \& Kosicki, 1993) beschreibt Berichtsstrukturen, zeichnet also nur Medien-Frames nach (Feld 6).

- Die öffentlichkeitstheoretische (u. a. Gerhards et al., 1998; Neidhardt \& Rucht, 1993; Weßler, 1999) und die bewegungstheoretische Perspektive (u. a. Gamson, 1992; Gamson \& Modigliani, 1989; Kliment, 1998; Snow \& Benford, 1988) sind makroskopisch ausgerichtet. Untersucht wird, welche politischen bzw. gesellschaftlichen Akteure ihre Bezugsrahmen (Feld 2) in den Medien lancieren können (Feld 5 und 6). Die Medien gelten dabei als „Transporteure“ für die Frames von Akteuren (Felder 2 und 8); zudem dienen Medieninhalte (Feld 6) als Indikator zur Erfassung öffentlicher Diskurse.

2 Am einfachsten lässt sich dies für Journalisten illustrieren (Scheufele, 2003: 91ff.): Journalistische Frames sind kognitive Erwartungsrahmen von Journalisten, die sich im redaktionellen und inter-mediären Diskurs herausbilden und verändern. Sie schlagen sich in der Berichterstattung als Medien-Frames nieder. 
- Die wirkungszentrierte Perspektive fragt danach, ob und wie die Rahmung eines Themas in den Medien (Feld 6) die Vorstellungen, Urteile, Bewertungen, Affekte und Entscheidungen von Rezipienten (Feld 7) beeinflusst. ${ }^{3}$

Zwischen den Perspektiven bestehen Überschneidungen. Erstens sind die MedienFrames inhaltszentrierter Studien letztlich wiederum eine unabhängige Variable der Wirkungsperspektive. Zweitens beleuchten öffentlichkeits- und bewegungstheoretische Arbeiten die Framing-Strategien öffentlicher Akteure, die unabhängige Variable der Kommunikatorperspektive sind. Drittens sind Journalisten und öffentliche Akteure ihrerseits Rezipienten von (Leit-)Medien. Darüber hinaus legt der Framing-Ansatz eher eine Mikro- bis Mesoperspektive an Journalismus an. Wir gehen davon aus, dass journalistische Frames in redaktionellen oder intermediären Gefügen entstehen und verändert werden. Damit hat der Framing-Ansatz andere Aussagebezüge als etwa die systemtheoretische Makroperspektive der jüngeren Journalismusforschung (z. B. Löffelholz, 2000).

\section{Terminologische Festlegungen}

Wir verfolgen nun die Wirkungsperspektive weiter. Dafür sind die Begriffe „Frame“ und „Framing“ zu klären. Wir schlagen eine Terminologie vor, die an psychologische und soziologische Grundlagen anknüpft, theoretisch und operational brauchbar sein soll und eine pragmatische Unterscheidung zwischen „Frame“ und „Schema“ ermöglicht (ausführlich Scheufele, 2003).

Die Kommunikationswissenschaft definiert „Frame“ oft analog zu „Schema“ (z. B. Entman, 1993: 52; Gitlin, 1980: 7; Valkenburg et al., 1999: 551). Damit wäre der FrameBegriff obsolet, da er nur an die Stelle etablierter Konstrukte wie „Schema“ (u. a. Higgins et al., 1981; Fiske \& Taylor, 1991; Brosius, 1991; Wicks, 1992) oder "Script“ (u .a. Rosch et al., 1976; Kleiber, 1998) treten würde. Wir behalten eingeführte Begriffe bei und integrieren das Frame-Konstrukt anders (Scheufele, 2003: 91, 94f.): Aus unserer Sicht beziehen sich kognitive Modelle (Schemata, Scripts u. ̈̈.) auf eine singuläre, spezifische Objektklasse (z. B. auf Politiker, auf den Ablauf einer Vorlesung) bzw. eine singuläre Relation zwischen Objekten (z. B. Ursachen-Schemata). Mehrere Schemata, Scripts usw. für verschiedene Bezüge spannen konsistente Sinnhorizonte bzw. Erwartungsrahmen auf. Diese kognitiven Frames betreffen also ein Bündel an Objektklassen und Relationen, d. h. einen ganzen Realitätsausschnitt (auch Scheufele, 1999a: 93; Scheufele, 2000: 383, 392).

Aus schematheoretischer Sicht mag man einwenden, dass auch Schema-Hierarchien ein abstraktes Schema in konkretere auffächern (z. B. Rogers \& Dearing, 1988; Yagade \& Dozier, 1990) und daher ein Frame nach unserer Konzeption ein Hauptschema sei. Dem ist dreierlei entgegenzusetzen: (1) Haupt- und Sub-Schemata beziehen sich auf dieselbe Objektklasse, während ein Frame sich aus Schemata für verschiedene Objektklassen bzw. Relationen (u. a. Ereignis, Akteur, Ursachen) konstituiert, die vergleichbare Maßstäbe anlegen. Eichhorns (1996: 85) eher weitgefasste Adaption von SchemaHierarchien für die Kommunikationswissenschaft ignoriert diesen wichtigen Unterschied. (2) Selbst wenn man ihn bestreitet, helfen Schema-Hierarchien nur bedingt weiter: Denn mit steigender Abstraktion erhält man ein einziges „Welt-Schema“, mit

3 Damit geht der wirkungszentrierte Ansatz über die rein kognitive Ebene (Feld 7) in Abbildung 1 hinaus. 
zunehmender Konkretisierung unendlich viele „Kleinst-Schemata“. Mit seiner rein theoretischen Erörterung, dass ein Thema „die Realisierung eines kognitiven Schemas [... ist], das eine Gruppe von Ereignissen, Handlungen, Interpretationen zusammenfaßt", entzieht sich Eichhorn (1996: 81) auch einer Antwort auf die Frage empirischer Prüfbarkeit. ${ }^{4}$ Zudem setzt er das Thema und die darauf bezogenen Kognitionen gleich. Dass zwischen beiden zu trennen ist, machen zwei einfache Überlegungen deutlich: Ein Frame kann auf verschiedene Themen angewendet werden und dasselbe Thema kann in verschiedene Frames gestellt werden (vgl. Scheufele, 2003: 97f.). (3) SchemaHierarchien verwischen die Grenzen zwischen der internen Struktur eines Schemas und der externen Verflechtung mehrerer Schemata für verschiedene Objektklassen. Diesen Problemen kann man nur mit einer pragmatischen Festlegung begegnen - etwa jener, die wir vorschlagen. ${ }^{5}$ Wir bestreiten nicht, dass sie ebenfalls Probleme aufwirft. Will man z. B. aus Antworten eines Rezipienten dessen kognitive Schemata ermitteln, so kann man deren Abstraktionsniveau nicht einfach theoretisch festlegen. Stattdessen sollte man z. B. den Allgemeinheitsgrad der häufigsten Aussagen in der Antwort des Rezipienten zu einer Objektklasse als Maßstab für sein Objekt-Schema wählen (vgl. zu konkreten Umsetzungsbeispielen u. a. Scheufele, 2001b, 2003: 137ff.). Auch der Objektbegriff lässt sich nur pragmatisch operationalisieren. Dabei wird man um Vorgaben nicht umhinkommen. Nur wenn man zuvor festlegt, dass man nach z. B. PersonenSchemata sucht, wird man aus den Antworten der Rezipienten etwas Sinnvolles - nämlich deren Personen-Schemata - extrahieren können.

Unsere Konzeption von Frames und Schemata integriert psychologische und soziologische Theorien, die bislang zu wenig berücksichtigt wurden:

Das schematheoretische Schablonenmodell (u. a. Tesser, 1978; Hastie, 1981) begreift ein Objekt-Schema als Konfiguration salienter Attribute, wobei diese als Leerstellen („slots“) mit typischen Ausprägungen („default values“) bzw. „Erwartungswerten“ (Schnotz, 1994: 62) gelten. Unser Basalmodell kognitiver Modelle (Schemata, Scripts u. ä.) unterscheidet zwischen Anwendungsbezug (Objekt bzw. Relation) eines Schemas und daran angelegten Maßstäben, die wir als Merkmalskonfiguration konzipieren (Scheufele, 2003: 92f.). So bezieht sich z. B. ein Täter-Schema auf die Objektklasse „Täter“, an die Maßstäbe wie z. B. „familiärer Hintergrund“ oder „psychische Disposition“ angelegt werden (Abbildung 2). Das Schablonenmodell ist operationalisierbar, weil es der Messlogik von Kategorie und Ausprägung folgt und die Identifizierung von Merkmalskonfigurationen in der Berichterstattung z. B. mit Hilfe von Faktoren- bzw. Clusteranalysen erlaubt. Zudem eignet es sich zur Identifizierung kognitiver Schemata. Denn es erlaubt den Einsatz von „Merkmalsräumen“ (Lazarsfeld \& Barton, 1951), d. h. Kombinationen von Kategorien und Ausprägungen, die ein zentraler Schritt der Idealtypenbildung im Sinne von Weber (1988) und Schütz (1993) sind. Die ermittelten Typen lassen sich als kognitive Schemata interpretieren. Denn Schemata und Idealtypen fokussieren gleichermaßen auf saliente Merkmale bzw. Maßstäbe und sehen gleichermaßen von „Nebensächlichem“ ab (vgl. Scheufele, 2003: 134ff.).

Auch gegen Schablonenmodell bzw. Schematheorie sind Einwände denkbar: (1) Die Schematheorie wurde wegen ihrer statischen Konzeption von Kognitionen kritisiert. Ansätze konnektionistischer Netzwerke (McClelland \& Rumelhardt, 1981, 1986;

4 So stellt sich die Frage, was denn „Realisierung“ konkret sein soll und wie sie zu messen wäre.

5 Vor einem vergleichbaren Problem steht übrigens auch die Nachrichtenwerttheorie mit dem Ereignisbegriff (vgl. dazu Staab, 1990: 100ff.). 
Dörffner, 1991) werden der Dynamik von Kognitionen zwar eher gerecht (Stoffer, 1990: 288), sind aber für kommunikationswissenschaftliche Sachverhalte kaum umsetzbar. (2) Die Existenz von Schemata sei nicht falsifizierbar, da man kaum Befunde finde, die sich nicht schematheoretisch erklären ließen (vgl. Alba \& Hasher, 1983; Fiske \& Taylor, 1991: 174ff.). Dieser Einwand lässt sich letztlich ebenso gegen andere sozialwissenschaftliche Konstrukte wie „Einstellung“ oder „Rolle“ vorbringen. Solange es nicht gelingt, z. B. konnektionistische Ansätze für komplexere Sachverhalte als Worterkennungen einfach zu operationalisieren, sind schematheoretische Überlegungen operational am brauchbarsten.

Abbildung 2: Basalmodell kognitiver Modelle (Schenfele, 2003)

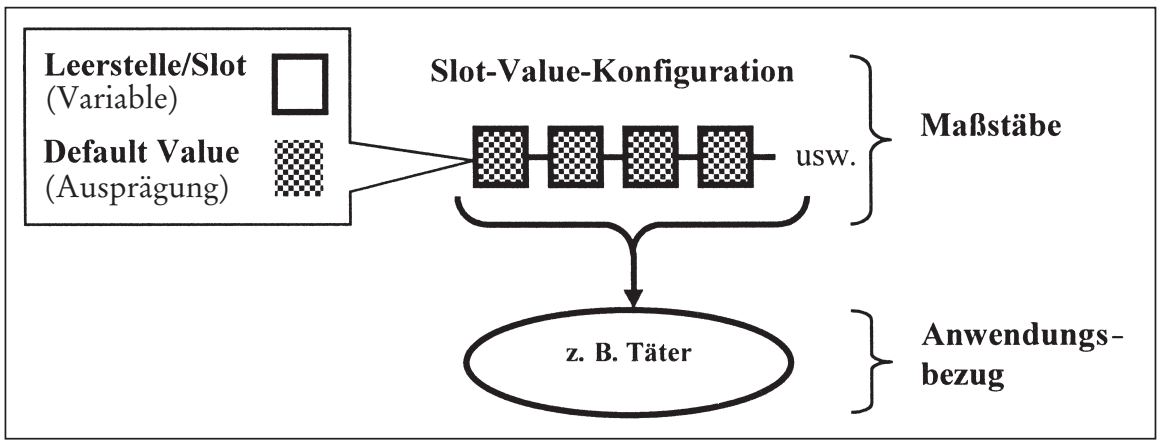

Der Gedanke, dass kognitive Modelle einen konsistenten Sinnhorizont bzw. Frame aufspannen, knüpft an psychologische Ansätze an: Einfache Netzwerktheorien (u. a. Lindsey \& Norman, 1977; Rumelhart \& Norman, 1978) konzipieren Wissen als Netzwerk aus einzelnen Knoten (Schemata) mit Verbindungen dazwischen, über die sich Aktivierung ausbreitet. Theorien konnektionistischer Netzwerke (McClelland \& Rumelhart, 1981, 1986; Dörffner, 1991) gehen von komplexeren Strukturen mit vielfältigen Aktivationswellen aus. Sie begreifen Wissen als dynamisches, anpassungsfähiges Aktivationsmuster in Netzwerken (Clark, 1989: 92f.). Aus dieser Sicht wäre ein kognitiver Frame nichts anderes als eine Konfiguration aus Schema-Knoten, die wiederholt aktiviert wurden, starke Verbindungsgewichte aufbauten und ein stabiles Aktivationsmuster bilden. Wenn Rezipienten z. B. ihr Schema für fremdenfeindliche Anschläge aktivieren, werden zugleich ihre Vorstellungen von „typischen“ Ursachen und Reaktionen aktiviert. Anschläge-, Ursachen- und Folgen-Schemata konstituieren dann einen gemeinsamen Sinnhorizont. Ähnliches kann man aus wissenssoziologischen und diskurstheoretischen Arbeiten ableiten. So spricht z. B. Lüders (1991: 383) von der „,inneren konsistenten Logik' von Deutungsmustern“. Öffentlichkeitstheoretische Ansätze (z. B. Neidhardt \& Rucht, 1993; Weßler, 1999) betonen ebenfalls, dass die Elemente kollektiver Frames (u. a. Problemdefinition, Ansprüche, Wertorientierungen) in einem Konsistenzverhältnis stehen. Vergleichbares impliziert die Rede von „interpretative packages“ (Gamson \& Modigliani, 1989) und einem „geschlossenen Sinnbereich“ (Schütz, 1971: 265) sowie der Gedanke der Erwartungsgrenzen sozialer Rahmen (Goffman, 1993: 62ff.).

Aus diesen Überlegungen ergibt sich unsere Konzeption von Framing: (1) Framing meint, bestimmte Objektklassen und Relationen eines Realitätsausschnitts zu betrachten und andere nicht. So können Journalisten z. B. entweder das Asylproblem oder die 
wachsende Zuwanderung für die Ursache fremdenfeindlicher Gewalt halten. (2) Framing meint aber auch, an die ausgewählten Objekttypen bestimmte Maßstäbe anzulegen und andere nicht. So können Journalisten z. B. als typische fremdenfeindliche Tat entweder Ausschreitungen vor Asylbewerberheimen oder Brandanschläge auf Gastarbeiterhäuser ansehen. Diese Konzeption geht über Entman (1993) hinaus: Denn erstens bleibt sein Begriff „aspects of perceived reality“ (Entman, 1993: 52) sehr vage und bezeichnet nur einen Teil von etwas. Zweitens spricht Entman (1993) zwar auch von „attributes“, fasst den Begriff aber eng. Unser Begriff „Maßstab“ ist also nicht etwa künstlich eingeführt, sondern umfasst weit mehr als „Attribut“ - nämlich neben Merkmalen von Objekten z. B. auch normative oder Wertbezüge. Drittens zählt Entman (1991) in seiner eigenen Studie nur Wörter und Etiketten aus, was seine Terminologie weiter einschränkt.

\section{Theoretisch-konzeptionelle Auseinandersetzung}

Die Auseinandersetzung mit der Wirkungsperspektive des Framing-Ansatzes wird in drei Schritten vollzogen: Zunächst erfolgt eine theoretisch-konzeptionelle Auseinandersetzung. Danach werden empirische Untersuchungen klassifiziert und methodisch wie empirisch diskutiert. Abschließend werden Probleme und offene Fragen der Forschung zu Framing-Effekten diskutiert.

\section{1 (Second-Level)-Agenda-Setting und Medien-Priming}

Berührungspunkte zwischen Framing, Agenda-Setting und Priming wurden mehrfach betont, wobei man zwei grundsätzliche Positionen unterscheiden kann: Die eine favorisiert Agenda-Setting als übergeordnetes Phänomen (u. a. Ghanem, 1997; Takeshita, 1997), die andere sieht Agenda-Setting als Variante von Priming und grenzt davon Framing ab (u. a. Price \& Tewksbury, 1997; Willnat, 1997). Beide Positionen sind kritikwürdig und unvollständig.

Nach der Agenda-Setting-Hypothese beeinflussen Medien nicht, wie Menschen denken, sondern worüber sie nachdenken (Cohen, 1963: 13). Neuerdings werden zwei Ebenen unterschieden (z. B. McCombs \& Ghanem, 2001).

- Agenda-Setting-Effekt: Die Gewichtung eines Objekts (Thema, Politiker) in den Medien beeinflusse die Bedeutung, die Rezipienten dem Objekt zuschreiben.

- Attribute-Setting-Effekt: Die Gewichtung kognitiver und affektiver Attribute des Objekts in den Medien beeinflusse die Bedeutung, die Rezipienten diesen Attributen zuschreiben.

Aus dieser Sicht bestimmen Medien nicht nur, über welche Objekte wir nachdenken, sondern auch wie wir über sie denken (Ghanem, 1997: 4). Frames werden als Attribute von Objekten und Framing als Selektion von Attributen verstanden (McCombs \& Ghanem, 2001: 74). Diese Überlegungen sind mehrfach kritikwürdig: (1) Denn bereits Benton \& Frazier (1976) oder Noelle-Neumann \& Mathes (1987) unterschieden zwischen „agenda-setting“ einerseits und „focussing“ bzw. „evaluating“ andererseits. (2) Zudem wird der Themenbegriff der Agenda-Setting-Hypothese nur durch einen Objektbegriff ersetzt. Der Objektbegriff ist zudem eingeschränkt, da empirische Studien (u. a. Golan \& Wanta, 2001; McCombs et al. (1997) nur nach politischen Kandidaten fragen. Eine Ausnahme machen zwar Jasperson et al. (1998), die Themen betrachten; allerdings zählen sie nur computergestützt Schlagwörter aus. Auch der Attributbegriff wird disparat konzipiert-als „perspective“, „frame“, „element“ oder „compelling argument“ (Gha- 
nem, 1997; McCombs \& Ghanem, 2001). (3) Daneben verkennt der Second-Level-Ansatz, dass affektive Attribute Tendenzen der Berichterstattung bzw. Einstellungen von Rezipienten betreffen. Damit ignoriert er die Forderung mehrerer Autoren (Brosius, 1995; Gamson \& Modigliani, 1989; Nelson et al., 1997; Scheufele, 2003), zwischen Kognition und Einstellung zu trennen. Dagegen mag man einwenden, Vorstellungen hätten stets auch affektive Bestandteile; zudem seien Kognition und Affekt seit den YaleStudies (z. B. Hovland et al., 1953; auch McGuire, 1985) als zwei der drei Komponenten von Einstellungen bekannt. Selbst dort werden sie aber analytisch sauber getrennt. (4) Mit unserem Verständnis von Framing ist der Ansatz konzeptionell nicht haltbar: Erstens sind Schemata spezifische Konfigurationen aus Attributen; Studien des SecondLevel-Ansatzes betrachten nur singuläre Attribute. Zweitens spannen Bündel an Schemata einen gemeinsamen Erwartungsrahmen auf, was ebenfalls verkannt wird (ähnlich Takeshita, 1997: 25). Drittens ist Framing nicht nur eine Funktion der Attribute (bzw. Maßstäbe), die an Objekte angelegt werden, sondern auch eine Funktion der Objekte selbst. (4) Schließlich fehlt jegliche Anknüpfung an die psychologische Forschung zu Attribute-Framing (dazu Levin et al., 1998), die exakt dasselbe wie der Second-Level-Ansatz untersucht.

Im Kontext von Agenda-Setting wird auch Medien-Priming diskutiert. Nach Iyengar \& Kinder (1987: 63) legen Medien, indem sie ein Thema hochspielen, zugleich die Kriterien fest, anhand derer Politiker beurteilt werden. Insofern kann man Medien-Priming als zweite Wirkungsstufe von Agenda-Setting begreifen (Iyengar \& Simon, 1993: 368; Pan \& Kosicki, 1997: 11). Iyengar \& Kinder (1987: 65) folgen der Verfügbarkeitsheuristik, wonach Menschen nicht alle, sondern nur aktuell zugängliche Kriterien für ihr Urteil heranziehen. Ein in den Medien präsentes Thema fungiere als Prime, der damit verknüpfte Kriterien bzw. Schemata zugänglich mache. Bei der späteren Urteilsbildung würden Rezipienten dann diese themenbezogenen Kriterien bevorzugen. ${ }^{6}$

Etwas anders stellt sich der ursprüngliche Ansatz in der Psychologie dar: (1) Eines der klassischen Experimente stammt von Ratcliff \& McKoon (1978). Sie ließen Probanden Sätze mit bestimmten Wörtern merken und vermuteten, dass sie dabei Wort-Assoziationen aufbauten. In einer Worterkennungsaufgabe sollten Probanden angeben, ob ein Target-Wort in einem Satz enthalten war. Der Experimentalgruppe wurde vor dem Zielwort ein Prime-Wort aus dem erlernten Satz eingeblendet. Entsprechend erfolgte die Erkennung hier schneller als in der Kontrollgruppe, was automatische Aktivationsausbreitung („,spreading activation“) zwischen Prime- und Target-Wort nahe legt (Collins \& Loftus, 1975). (2) Higgins et al. (1977) oder Wyer \& Srull (1981) gehen von einem Link zwischen Eigenschaften („traits“) und Verhalten aus: Werden Probanden auf Traits geprimet, klassifizieren sie das Verhalten einer Zielperson (Target) im Sinne des Trait-Primes. Dabei muss das Verhalten mehrdeutig und der Prime anwendbar sein; zudem darf Menschen der Link nicht bewusst sein, damit keine Kontrasteffekte auftreten (Lombardi et al., 1987). (3) Die abhängige Variable ist meist die Klassifizierung von Verhalten durch Nennung einer Eigenschaft (Higgins et al., 1985); untersucht werden aber auch Problemlösen (Higgins \& Chaires, 1980) oder Persuasion (Bohner et al., 1995). (4) Priming-Effekte sind umso stärker, je kürzer der Prime zurückliegt (Higgins et al., 1985) bzw. je häufiger Priming erfolgte (Srull \& Wyer, 1979). Ob Recent oder Frequent Priming dominiert, wird unterschiedlich gesehen: Nach dem Storage-Bin-Modell (Wyer

6 Peter (2002: 23) will entdeckt haben, dass Priming zwei Schritte umfasst. Tatsächlich wurden beide Schritte schon von anderen Autoren (z. B. Pan \& Kosicki, 1997: 11) herausgearbeitet. 
\& Srull, 1981) ist das Gedächtnis ein „kognitiver Eimer“; kürzlich geprimete Schemata liegen oben, können also leichter hervorgeholt werden. Nach dem Storage-Battery-Modell (Wyer \& Carlston, 1979) wird die „Batterie“ von Schemata durch häufiges Priming wiederholt aufgeladen, so dass sie leichter zugänglich sind. Nach dem Synapsen-Modell (Higgins et al., 1985) nimmt der Erregungslevel bei kürzlich geprimeten Schemata schneller ab. Wenn zwischen Prime und Target wenig Zeit verstreicht, dominiert daher Recent Priming, bei längerer Zeitspanne dagegen Frequent Priming. Empirische Befunde sprechen am ehesten für das Synapsen-Modell (Higgins, 1989).

Ungeachtet zahlreicher empirischer Belege für Medien-Priming ist die Übertragung des psychologischen Priming-Konzepts auf die Kommunikationswissenschaft problematisch:

- Medien-Priming-Studien unterstellen, dass Themen so wirken wie singuläre Wörter oder Eigenschaften. Dabei hat Berichterstattung eine weit komplexere Struktur und kann daher mehrfach oder sogar widersprüchlich primen. ${ }^{7}$ Hier wird also eine These zur Wirkung von Mikrostimuli auf Makrostimuli und damit die falsche Analyseebene übertragen. Ein Frame als Bündel aus Ereignis-, Akteurs-, Kausal- und Finalvorstellungen bzw. entsprechenden Darstellungen entspricht im Vergleich dazu viel eher der Struktur und Komplexität von Medienbeiträgen.

- Experimente zu Medien-Priming werden tatsächlicher Medienrezeption im Grunde nicht gerecht. Priming-Effekte können letztlich nur bei kumulativer und konsonanter Berichterstattung über ein Thema eintreten. Ein Längsschnittdesign (Willnat \& Zhu, 1996) bzw. sequenzielle Experimente, die den Prime-Stimulus an mehreren aufeinander folgenden Tagen induzieren (Iyengar \& Kinder, 1987), werden aber nur selten realisiert.

\subsection{Schema-Aktivierung durch Medien-Framing und Medien-Priming}

Für ihre Abgrenzung von Agenda-Setting, Priming und Framing folgen Price \& Tewksbury (1997; Price et al., 1997) der Unterscheidung in Verfügbarkeit, Zugänglichkeit und Anwendbarkeit von Schemata (Higgins, 1989; Higgins \& Brendl, 1995). Verfügbarkeit (,availability“) meint, dass ein Schema im Langzeitgedächtnis gespeichert ist. Anwendbarkeit („applicability“) meint, dass sich Schlüsselreize einer Information mit salienten Schema-Slots decken. Zugänglichkeit („accessibility“) meint, dass das Schema in den Arbeitsspeicher gelangt und damit der Zugriff darauf wahrscheinlicher ist bzw. schneller erfolgt. Meist wird zwischen dauerhafter und kurzfristiger Zugänglichkeit unterschieden. Ursache dafür sind kurzfristiges oder wiederholtes Priming.

Als gemeinsame Klammer von Agenda-Setting, Priming und Framing gilt SchemaAktivierung: (1) Bei Framing fungierten saliente Merkmale der Medienbotschaft als Schlüsselreize, die jene Schemata aktivieren, deren Slots sich mit den Reizen am ehesten decken. Dieses „Fitting“ (Scheufele, 1999a: 94f.) begreifen die Autoren als Frage der Anwendbarkeit von Schemata. Der Framing-Effekt sei daher ein „applicability-effect“, der während oder unmittelbar nach der Rezeption auftrete (Price et al., 1997: 486). (2) Wenn ein Schema durch Framing aktiviert werde, behalte es für gewisse Zeit ein residuales Erregungsniveau. Daher sei die Wahrscheinlichkeit hoch, dass es bei einem späteren Urteil wieder aktiviert werde. Die Aktivierung des Schemas sei dann ein „accessibility-effect“,

7 Zudem ist zu vermuten, dass Medien ihre Rezipienten nicht nur auf ein Thema, sondern auch auf Bewertungen primen (Pan \& Kosicki, 1997: 10f.). 
der sich durch Priming erklären lasse. In unseren Worten: Langfristig werden Vorstellungen, Bewertungen und Entscheidungen von Rezipienten dadurch beeinflusst, dass Medien einerseits kumulativ primen, indem sie wiederholt prominent über ein Thema berichten, und andererseits dieses Thema auf stets gleiche Weise darstellen, es also konsonant framen. ${ }^{8}$ Auch wenn Price \& Tewksbury (1997) den bislang überzeugendsten Abgrenzungsversuch der drei erwähnten Wirkungskonzepte liefern, weist ihr Modell zwei zentrale Defizite auf: Erstens kann es nicht die Veränderung bestehender bzw. die Etablierung neuer Vorstellungen bei Rezipienten erklären. Zweitens bleibt offen, weshalb die Aktivierung bestimmter Schemata zu bestimmten Urteilen führt.

\subsection{Veränderung und Etablierung von Vorstellungen und Meinungen}

Um die Veränderung bisheriger und die Etablierung völlig neuer Schemata zu erklären, bieten sich Theorien mentaler Modelle (Johnson-Laird, 1983, 1989) oder situationaler Modelle (van Dijk, 1988) an (Scheufele, 2003: 22ff., 65ff.): Solche Modelle werden als Repräsentationen der z. B. in einem Zeitungsbeitrag erwähnten Situation verstanden: Wer einen Artikel über den Nahostkonflikt liest, hat schon ein Bild von der dortigen Situation. Bei der Lektüre wird es aber kontinuierlich validiert, erweitert oder neu konstruiert. Modellkonstruktion ist ein Zusammenspiel aus drei Komponenten: dem präsenten Teil der Textbasis (Berichterstattung), dem momentan aktivierten Vorwissen (Schemata) und dem aktuell konstruierten Modell. Auch mentale Modelle haben Leerstellen, die man als die offenen Fragen des Lesers an den Zeitungsartikel sehen kann. Diese werden mit Informationen aus dem Artikel oder mit schemaspezifischen Erwartungswerten besetzt. So bringen Rezipienten ihr Wissen auf den neuesten Stand (van Dijk \& Kintsch, 1983: 342).

Übertragen auf Framing bedeutet dies (Scheufele, 1999a: 100ff.): In der Berichterstattung präsente Bezugsrahmen und vorhandene Rezipienten-Schemata spielen auf spezifische Weise zusammen. Dabei halten wir drei Fälle für relevant (Abbildung 3).

- Fall 1 - Transformation bestehender Schema-Maßstäbe: Durch kumulative und konsonante Berichterstattung werden Vorstellungen von Rezipienten (Kreis in Abbildung 3) sukzessive verändert (Oval), bis sie sich der Medienrealität angeglichen haben (Rechteck).

- Fall 2 - Veränderung bestehender relationaler Vorstellungen: Relationale Vorstellungen z. B. über die Ursachen von Geschehnissen kann man als Kausalverbindung zwischen Wissensknoten modellieren. Wenn Medien kumulativ bestimmte Erklärungen anführen, werden Rezipienten immer weniger ihre bisherige Kausalattribution (Kreis) und immer stärker die medial vermittelte Kausalattribution vornehmen (Rechteck).

- Fall 3 -Etablierung neuer Vorstellungen: Rezipienten verfügen nicht immer über ein spezifisches Schema z. B. für fremdenfeindliche Gewalt und ziehen daher ihr generelles Gewalt-Schema heran. Mit dem Subtyping-Modell der Schematheorie (Fiske \& Taylor, 1991) dürften das Gewalt-Schema und die mediale Rahmung fremdenfeindlicher Gewalt aber zur Herausbildung eines neuen Sub-Schemas für fremdenfeindliche Gewalt führen.

8 Kumulative und konsonante Berichterstattung versteht Noelle-Neumann (1973; Noelle-Neumann \& Mathes, 1987) als zentrale Bedingung starker Medienwirkung. Vergleichbares besagt die Kultivierungsthese (u.a. Gerbner et al., 1999; Signorelli \& Morgan, 1990). 
Fall 1: Anschläge-Framing und Transformation des Anschläge-Schemas (Veränderung von Maßstäben / Attribut-Konfigurationen)

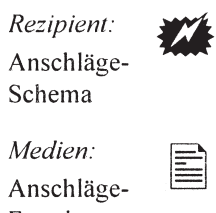

Framing

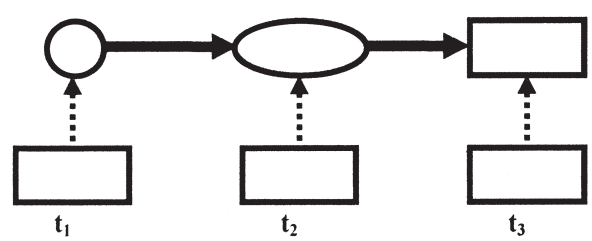

$\mathbf{t}_{\mathbf{3}}$

Fall 2: Kausales Framing und Transformation von Kausalvorstellungen (,Verlegung' der kausalen Verbindung)

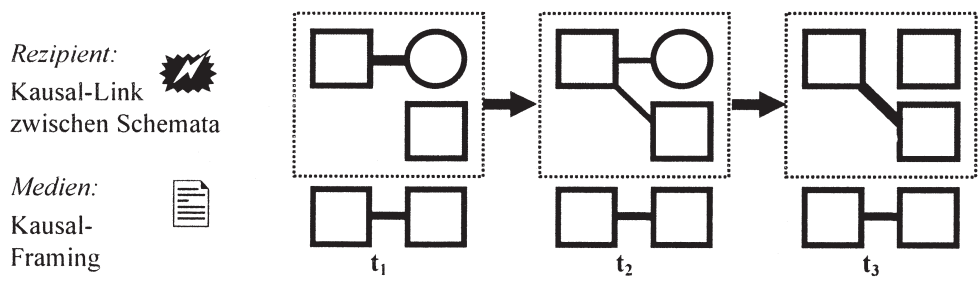

Fall 3: Medien-Framing und Etablierung eines spezifischen Sub-Schemas Rezipient:

Etablierung eines Sub-Schemas

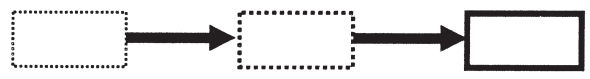

Medien:

Medien-

Framing

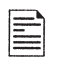

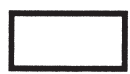

$\mathbf{t}_{1}$

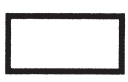

$t_{2}$

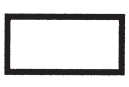

$\mathbf{t}_{3}$

Transformation

$\square \bigcirc$ Rezipienten-Schemata

Diese Unterscheidung ist analytischer Natur. In der „Realität“ dürften die Effekte konfundieren. So wird man in einem Experiment z. B. nicht nur eine Veränderung des Ereignis-Schemas, sondern zugleich auch eine Veränderung von Kausalvorstellungen finden.

Der Framing-Ansatz postuliert, dass die Aktivierung eines Schemas auf der ersten Stufe auch bestimmte Bewertungen, Entscheidungen oder Affekte auf der zweiten Stufe nahe legt. Dies lässt sich als „schema-triggered affect“ Fiskes (1982) oder kognitiv-affektive Inferenz (Peeters, 1991) beschreiben. (1) Eine vergleichbare Erklärung gibt die Wirkungsperspektive der Theorie instrumenteller Aktualisierung (Kepplinger et al., 1991), die sich gar nicht auf Framing-Effekte bezieht. Mit Rosenbergs (1956) Theorie affektiv-kognitiver Konsistenz haben Menschen eine positive Einstellung zu einem Einstellungsobjekt, wenn es für zentrale Werte instrumentell nützlich ist (positive Kogni- 
tion). Je mehr positive (negative) Kognitionen bestehen, desto positiver (negativer) ist die Einstellung zum Sachverhalt. Die Wirkung der Medien besteht darin, dass sie bestimmte Sachverhalte hochspielen und diese für Rezipienten zunächst salienter machen. Im nächsten Schritt gleichen Rezipienten ihre Einstellung diesen salienten Kognitionen an, um kognitiv-affektive Konsistenz herzustellen. (2) Explizit auf Framing beziehen sich Nelson et al. (1997): Das klassische Einstellungskonzept nehme an, dass Einstellungen verändert werden, indem Medienbotschaften neue Informationen (Argumente) vermitteln. Framing beeinflusse dagegen nur bestehende Vorstellungen. Nach dem Erwartungs-Bewertungs-Modell (Fishbein \& Ajzen, 1975) erwarten Menschen, dass ein Objekt bestimmte Eigenschaften aufweist (kognitive Komponente). Die Erwartung kann als Gewicht der Eigenschaft verstanden werden. Jede Eigenschaft wird zudem bewertet (affektive Komponente). Die Einstellung ergibt sich als Summe der Produkte aus Gewichten und Bewertungen der Objektmerkmale. Daraus leiten Nelson et al. (1997: 225f.) eine Erklärung für Framing-Effekte ab, die sich an folgendem Beispiel illustrieren lässt: Ein Rezipient erwartet von Kernkraft neue Arbeitsplätze, aber auch wachsende Entsorgungsprobleme. Da beide Eigenschaften gleiches Gewicht haben, ist seine Einstellung ambivalent. Wenn Medien nun Kernenergie wiederholt in den Bezugsrahmen von Entsorgungsproblemen stellen, machen sie diese Eigenschaft salienter. Dadurch erhält sie mehr Gewicht in der Produktsumme. Zugleich geht die damit verbundene Bewertung stärker in die Einstellungssumme ein, die insgesamt negativer wird. Allerdings kann Medien-Framing unseres Erachtens nur dann Breitenwirkung entfalten, wenn die meisten Rezipienten die betreffenden Eigenschaften auch de facto vergleichbar bewerten.

Zusammenfassend sehen wir vier Typen von Framing-Effekten: Medien-Framing aktiviert Schemata (Aktivierungs-Effekt). Kumulativ und konsonant führt es dazu, dass diese wiederholt aktiviert werden und für Urteile leicht zugänglich sind (Priming-/Zugänglichkeits-Effekt). Kumulatives, konsonantes Framing kann Rezipienten-Schemata aber auch in Richtung des Medien-Frames verändern (Transformations-Effekt) oder überhaupt erst zur Herausbildung eines (Sub-)Schemas führen (Etablierungs-Effekt). ${ }^{9}$ Schließlich verändert mediales Framing die kognitive und damit die affektive Komponente von Einstellungen bzw. Meinungen der Rezipienten (Einstellungs-Effekt).

Tabelle 1: Typen von Framing-Effekten (Scheufele, 2003)

\begin{tabular}{lll}
\hline Bezeichnung & Beschreibung & Mechanismus \\
\hline Aktivierungs-Effekte & $\begin{array}{l}\text { Aktivierung bestehender Schemata } \\
\text { Wiederholte Aktivierung (Priming) }\end{array}$ & $\begin{array}{l}\text { Kurzfristige Anwendbarkeit } \\
\text { Langfristige Zugänglichkeit }\end{array}$ \\
\hline $\begin{array}{l}\text { Transformations- } \\
\text { Effekt }\end{array}$ & $\begin{array}{l}\text { Veränderung bestehender Schemata } \\
\text { in Richtung des Medien-Frames }\end{array}$ & $\begin{array}{l}\text { Konsonante und kumulative } \\
\text { Berichterstattung }\end{array}$ \\
\hline Etablierungs-Effekt & $\begin{array}{l}\text { Etablierung nicht vorhandener } \\
\text { Schemata bzw. Schema-Links }\end{array}$ & u. a. Subtyping von Schemata \\
\hline Einstellungs-Effekt & $\begin{array}{l}\text { Veränderung bestehender } \\
\text { Einstellungen und Meinungen }\end{array}$ & $\begin{array}{l}\text { Veränderung affektiver } \\
\text { durch Veränderung kognitiver } \\
\text { Einstellungskomponenten }\end{array}$ \\
\hline
\end{tabular}

9 Das Zusammenspiel von Medienbotschaft und Rezipientenwissen beschreibt in ähnlicher Form auch das dynamisch-transaktionale Modell (Früh \& Schönbach, 1982; Früh, 1991a). 


\section{Methodisch-empirische Auseinandersetzung}

Auf die theoretisch-konzeptionelle folgt nun eine methodisch-empirische Auseinandersetzung. Die dafür ausgewählten Studien klassifizieren wir im ersten Schritt nach unabhängigen Variablen, im zweiten Schritt nach abhängigen Variablen, also nach den vier eben erwähnten Framing-Effekten.

\subsection{Unabhängige Variablen - Effekte unterschiedlicher Medien-Frames}

Bezogen auf unabhängige Variablen lassen sich die Studien in zwei Klassen einteilen: Die erste beleuchtet die Wirkung formal-abstrakter, die zweite die Wirkung inhaltlicher Medien-Frames.

\subsubsection{Effekte formal-abstrakter Frames}

Als formal-abstrakt bezeichnen wir Medien-Frames, die nicht auf inhaltliche Aspekte abheben. Solche Stimuli sehen wir als Entsprechung des schematheoretischen Konzepts inhaltsfreier, abstrakter Schemata (z. B. „Kausalität“ oder „Balance“; vgl. Axelrod, 1973; Kelley, 1972).

Iyengar (1991: 13ff.) unterscheidet „episodic“ und „thematic framing“. Im ersten Fall konzentriere sich die Berichterstattung auf einzelne Ereignisse, die als charakteristische Beispiele dienen; dies erinnert an Prototypen und Fallbeispiele (Daschmann, 2001). Im zweiten Fall werde ein mehr oder minder abstrakter thematischer Zusammenhang hergestellt. Die abhängige Variable ist die Verantwortungszuschreibung für Probleme und deren Lösung. Bei episodischer Darstellung werde Individuen bzw. Betroffenen, beim thematischen Frame der Gesellschaft bzw. den Umständen die Verantwortung zugewiesen. Iyengar (1991: 11ff.) wählte einen Mehr-Methoden-Ansatz: Mit einer Inhaltsanalyse wurden TV-Nachrichten auf Framing untersucht. In Feldexperimenten wurden Beiträge aus den TV-Nachrichten im episodischen bzw. thematischen Modus vorgespielt und Fragen zur Verantwortungszuschreibung gestellt. Umfragedaten gaben Aufschluss über weiter gehende Effekte. Die Befunde aller Experimente lassen sich hier nicht diskutieren. Insgesamt schienen sie die Annahmen zu bestätigen. Allerdings zeigte z. B. ein Experiment zu Armut (Iyengar, 1991: 46ff., 53ff., 59ff.), dass nicht nur das Framing von Armut die Richtung beeinflusste, in der Probanden Verantwortung zuschrieben, sondern auch die Art der Opfer. Bei Arbeitslosigkeit blieben Effekte völlig aus, was ein Deckeneffekt sein dürfte (dazu Kepplinger et al., 1989: 147). Diskussionswürdig sind die Befunde zu Rassendiskriminierung und Armut (Iyengar, 1991: 65ff.), weil sie unseres Erachtens auf die Konfundierung des Stimulus mit anderen Beitragsmerkmalen zurückgehen.

Inhaltsanalyse- und Umfragedaten kombinierte die theoretisch vergleichbare Studie von Iyengar \& Simon (1993). Eine Inhaltsanalyse zeigte, dass die Fernsehbeiträge über die Golf-Krise 1990/91 meist episodisch waren und sich ihr Schwerpunkt gegen Ende des Untersuchungszeitraums auf eine militärische Intervention verlagerte. Eine Bevölkerungsumfrage ermittelte die Zustimmung zu einer militärischen bzw. diplomatischen Intervention. Befragte mit starkem Fernsehkonsum bevorzugten eher eine Militärintervention. ${ }^{10}$ Allerdings wäre dies nur dann ein Medien-Effekt, wenn die Mehr-

10 Dies stützt sich auf Iyengars (1991) Experimente zu Kriminalität: Probanden favorisierten bei episodischem Framing eine Bestrafung und machten bei thematischem Framing die Gesellschaft verantwortlich. 
heit episodischer Beiträge tatsächlich eine militärische Option anspricht; das Gegenteil war der Fall. Zudem hatte die Verlagerung der Berichterstattung auf eine militärische Lösung und die wachsende Zustimmung der Bevölkerung schlicht mit der realen Intervention der USA in Kuwait zu tun. Diesen Scheinzusammenhang übersehen die Autoren.

Rhee (1997) greift auf Pattersons (1993) These zurück, dass Medien Politik zunehmend als strategischen Wettkampf darstellen: Beim „strategic frame“ behandelten sie Strategien, Taktiken und Persönlichkeit von Kandidaten, beim „issue frame“ dagegen Sachthemen. Als Framing-Effekt galt die Verschiebung der Rezipientenvorstellungen in Richtung des Frames, womit Rhee (1997: 28f.) Überlegungen zu mentalen Modelle aufgriff. Im Feldexperiment war der Frame ein erster, die Mediengattung ein zweiter und das Vorwissen der Probanden ein dritter Faktor. Ein Pre- und ein Posttest erfassten Rezipientenvorstellungen („narrative representations of campaign interpretations“; Rhee, 1997: 35). Varianzanalysen zeigten, dass Rezipienten strategischer (thematischer) Beiträge häufiger eine strategische (thematische) Perspektive an die Wahl anlegten als die andere Experimentalgruppe. Zudem waren Probanden, die ohnehin in der jeweiligen Kategorie dachten, anfälliger für den jeweiligen Frame; signifikante Belege fanden sich aber nur für das Printformat.

Rhees (1997) Studie entstand im Kontext eines Projekts von Cappella \& Jamieson (1996, 1997). Der erste Projektteil betraf eine Bürgermeisterwahl, der zweite die Gesundheitsreform Clintons. Als Stimuli der Feldexperimente wurden Kombinationen des strategischen und thematischen Framing variiert - teilweise geknüpft an die Mediengattung. Abhängige Variablen waren u. a. Kenntnisse der Sachverhalte, politische Einstellungen sowie Politikverdrossenheit; Cappella \& Jamieson (1997: 141ff.; 209ff.) sprechen von „political cynicism“ und meinen damit den Vertrauensverlust in politische Institutionen und Medien. Die Stimulusgabe erfolgte an mehreren aufeinander folgenden Tagen. Nur die Experimente zur Bürgermeisterwahl schienen die Vermutung zu bestätigen, dass strategiebezogene Berichterstattung Politikverdrossenheit fördert. Für die Experimente zur Gesundheitsreform boten Cappella \& Jamieson (1997: 160ff; 1996: 81ff.) zwar mehrere Erklärungen. Aus unserer Sicht wurde der Stimulus aber durch die tatsächliche Rezeption der Debatte um die Reform überlagert. Zudem wurde der IssueFrame zu problembezogen definiert. Insgesamt legen die Befunde keineswegs eine Politikverdrossenheit durch „strategic framing“ nahe.

Die Wirkung von „value-framing“, den Übergang von formal-abstraktem zu inhaltlichem Medien-Framing, untersuchten Shah et al. (1996, 2001). Unterschieden wurde Framing aus ethisch-moralischer und aus materialistischer Grundhaltung. Die abhängige Variable war die Entscheidung für einen Kandidaten. Sie galt als kompensatorisch, wenn Probanden den Kandidaten aufgrund seiner Positionen zu mehreren Themen auswählten; bei nur einem Kriterium galt sie als nicht-kompensatorisch. Rezipienten des moralisch bzw. ethisch argumentierenden Artikels legten häufiger ethische Standards an als die Rezipienten des materialistisch argumentierenden Beitrags. Zudem kamen sie häufiger zu einer nicht-kompensatorischen Entscheidung.

\subsubsection{Effekte inhaltlicher Frames}

Inhaltliche Frames stellen einen Sachverhalt in einen inhaltlichen Sinnzusammenhang, statt ihn aus der Warte eines allgemeinen Prinzips (z. B. „Balance“) zu betrachten oder auf formale Weise (z. B. episodisch) zu präsentieren. Ein formaler Frame würde einen Terror-Anschlag z. B. episodisch oder thematisch präsentieren, während bei inhaltli- 
chem Framing z. B. ein „Nahost-Frame“ oder ein „Religiöser Fanatismus-Frame“ angelegt wird - ob nun in episodischer oder thematischer Form.

Neuman et al. (1992) untersuchten neben anderen Fragen jenseits von Framing auch die Übereinstimmung von Medien- und Rezipienten-Frames. Die Transkripte von 43 Tiefeninterviews diskutierten Codierer im Hinblick auf erkennbare Frames. Diese Frames dienten dann als Kategorien einer Inhaltsanalyse. Der Vergleich der Anteile von Frames bei Medien und Befragten ergab unterschiedliche Gewichtungen, die aber aus methodischen Gründen wenig aussagen. Ein Experiment von Valkenburg et al. (1999) unterschied vier Gruppen mit je einem Frame sowie eine Kontrollgruppe. Jede Gruppe erhielt zwei Zeitungsbeiträge zu Kriminalität und Euro. Die Probanden sollten ihre Gedanken und Gefühle äußern („thought-listing“; vgl. Price et al., 1997). Bei der Codierung der Antworten der Rezipienten mussten Codierer Fragen zu den vier Frames beantworten. Die "Codierungen“" wurden faktorenanalytisch gebündelt und die Faktoren als Frames interpretiert. Hier stellt sich die Frage, ob nun die vorab festgelegten Frames oder die Faktoren als Frames gelten sollen. Multivariate Varianzanalysen zeigten, dass der jeweilige Medien-Frame die Gedanken der Probanden beeinflusste. Problematisch sind die ausgewählten Frames; dasselbe betrifft Neuman et al. (1992), denen Valkenburg et al. (1999) folgten, sowie Price et al. (1997). Alle drei Studien wählten „conflict“, „consequences“ oder "human interest“ als inhaltliche Frames. Worin sich diese Frames von Nachrichtenfaktoren unterscheiden sollen, lassen die Autoren jedoch offen.

Eine überzeugendere Operationalisierung inhaltlicher Frames bietet ein Experiment zur „Iran-Contra-Affäre“ von Iyengar (1991: 69ff.). Eine Stimulusvariante rahmte die Affäre z. B. vor dem Hintergrund der Glaubwürdigkeit Reagans und seiner Kenntnis der Affäre. Die Probanden wurden nach den Gründen des Waffenverkaufs gefragt. Die Befunde zeigten starke, teils signifikante Unterschiede zwischen den Gruppen. Befunde nationaler Umfragen wiesen in dieselbe Richtung, belegten aber einen klaren parteipolitischen Bias. Auch Park \& Kosicki (1995) untersuchten inhaltliches Framing bei der Iran-Contra-Affäre. Sie unterschieden zwei Klassen von Frames: Bei „valence issues“ (z. B. Reagans Inkompetenz) konnte man nur eine negative, bei „position issues“ (z. B. Waffen für Libanon-Geiseln) dagegen verschiedene Meinungen haben. Die sinkende Zustimmung zu Reagan mit wachsender Mediennutzung erklärten die Autoren mit der Dominanz negativer Valenz-Frames, die eine Inhaltsanalyse der TV-Nachrichten erbracht hatte. Diese Folgerung beruht aber nur auf Plausibilität, weil Inhaltsanalyse und Befragung nicht verknüpft wurden.

Ein Experiment von Nelson et al. (1997) vermittelte im ersten Stimulusartikel, dass Sozialhilfe jene Menschen erhielten, die Hilfe nicht verdienten („give-away-program“). Der andere Artikel rahmte Sozialhilfe als Belastung für die Wirtschaft. Die Autoren vermuteten, dass der erste Frame Armutsvorstellungen aktiviert, die mit Einstellungen zur Sozialhilfe korrelieren. Beim Wirtschafts-Frame dürfe keine solche Korrelation bestehen. Als Framing-Effekt galt der Unterschied der Korrelationswerte zwischen beiden Gruppen. Die Befunde zeigten, dass die Korrelation zwischen der Haltung zu Sozialhilfeempfängern und den Einstellungen zur Sozialhilfe beim Frame „give-away-program“ stärker war als beim Wirtschafts-Frame. Zudem war die Korrelation bei Menschen mit viel und wenig Argumentkenntnis vergleichbar hoch. Dies spreche nur für eine Veränderung des Gewichts bestimmter Informationen und damit für einen Framing-Effekt. 


\subsection{Abhängige Variablen - Typen von Framing-Effekten}

Empirische Studien klassifizieren wir nun nach den abhängigen Variablen und damit nach dem Effekttyp, den sie beleuchten. Besprochen werden dabei weitere Arbeiten, die einen der Effekte beleuchten, ohne das Framing-Etikett zu bemühen.

\subsubsection{Transfer von Medien-Frames und Aktivierung von Rezipientenvorstellungen}

Die Übernahme von Medien-Frames durch Rezipienten untersuchten Neuman et al. (1992) und Arbeiten zu Attribute-Agenda-Setting (z. B. Golan \& Wanta, 2001; McCombs et al., 1997). Letztere korrelierten auf Aggregatdatenniveau die Häufigkeit der Nennung von Attributen in der Berichterstattung und in Umfragen bei Wahlkämpfen. Gegen die Studien lässt sich ähnliche Kritik wie gegen die Chapel-Hill-Studie (McCombs \& Shaw, 1972) vorbringen: So bleibt die Kausalrichtung völlig unklar und die Korrelationshöhe geht auf das Aggregatdatenproblem, also einen ökologischen Fehlschluss zurück (dazu Rössler, 1997: 97). ${ }^{11}$ Die erwähnten Arbeiten prüften zudem nur die Übereinstimmung von Medien- und Rezipienten- „Frames“, ohne zu klären, ob diese auf eine Aktivierung oder Transformation bestehender Rezipienten-Schemata zurückgeht. Damit suggerieren sie, dass Rezipienten die in der Berichterstattung präsenten inhaltlichen Frames direkt übernehmen. Framing-Effekte sind aber kein simpler Transfer von Medien-Frames in die Vorstellungswelt der Rezipienten, sondern das Ergebnis einer Interaktion aus beiden (vgl. Scheufele, 1999a: 100ff.; auch Johnson-Laird, 1983, 1989; Früh, 1991b: 241ff.).

Explizit auf den Aktivierungs-Effekt beziehen sich Price et al. (1997). Ein erstes Experiment unterschied zwei Experimental- und eine Kontrollgruppe. Die Probanden sollten nach der Lektüre frei ihre Gedanken äußern. Diese folgten dem Medien-Frame: So äußerten sich z. B. Leser des Artikels mit Konflikt-Frame häufiger konfliktbezogen als die übrigen Probanden, was die Autoren als Beleg für die Aktivierung bestimmter Kognitionen interpretierten. Auch die schon vorgestellten Experimente von Valkenburg et al. (1999) und Nelson et al. (1997) beleuchteten eine Aktivierung von Rezipientenvorstellungen, wiesen diesen Effekt aber nur indirekt nach.

\subsubsection{Transformation und Etablierung von Rezipientenvorstellungen}

Zur Etablierung neuer und Veränderung bestehender Vorstellungen von Rezipienten gibt es so gut wie keine Framing-Studie. Hinweise stammen aber aus Studien zur Informationsvermittlung.

Früh (1991b) untersuchte, ob Rezipienten durch die Reihenfolge von Informationen in einem Zeitungsartikel einen Sinnzusammenhang generieren, der die Lesart für die weitere Rezeption vorgibt. Unterschieden wurden drei Versionen eines Artikels über Produktionsstockungen, die alle dieselben Informationen enthielten - nur in anderer Reihenfolge. Die Erklärungen folgten tatsächlich dem durch die Informationsreihenfolge etablierten Sinnhorizont. Dadurch wurden selbst Informationen am Ende des Artikels, die das Gegenteil nahe legten, gar nicht wahrgenommen.

11 Zudem wurden weder bestehende Schemata noch Mediennutzung erfasst. Auch orientierten sich die codierten Merkmale an den Umfragen, was die falsche Analyserichtung ist (dazu Kepplinger et al., 1989: 77). 
Auch ein Experiment von Staab (1992) zu Ausstrahlungseffekten ist keine originäre Framing-Studie. Untersucht wurde der Einfluss einer Wirtschaftsmeldung aus Ostdeutschland zu Beginn einer TV-Nachrichtensendung auf die Erinnerung an eine Meldung über Rechtsradikalismus am Sendungsende. Hatte die Wirtschaftsmeldung negativen Tenor, erinnerten sich Probanden häufiger an die spätere Meldung. Den Effekt kann man so erklären: Bei negativem Tenor verknüpften Probanden beide Meldungen, indem sie die Wirtschaftslage als Ursache für Rechtsradikalismus attribuierten. Hier schien also eine kognitive Kausalrelation etabliert worden zu sein.

Explizit auf Framing beziehen sich Brosius \& Eps (1995). Sie konfrontierten inhaltsanalytische Daten, Befragungsdaten und Realitätsindikatoren. Die Befragten sollten ihre Vorstellungen von sechs Merkmalen fremdenfeindlicher Taten angeben. Die Operationalisierung folgte dem Kultivierungsansatz; so sollten die Befragten z. B. einschätzen, wie häufig es Brandanschläge, Angriffe auf Personen und sonstige Taten gibt. In der Tat überschätzten die Befragten z. B. den Anteil von Brandanschlägen im Vergleich zu deren tatsächlichem Ausmaß. Brosius \& Eps (1995: 175) weisen darauf hin, dass man Rezipientenvorstellungen nach deutlichen Veränderungen in der Berichterstattung bzw. Ereignislage (z. B. nach den Brandanschlägen in Mölln oder Solingen) jeweils neu erheben müsse, was retrospektiv nicht möglich war. Damit erwähnen sie implizit Transformations-Effekte. Weniger leuchtet dagegen die am Kultivierungsansatz orientierte Operationalisierung von Schemata ein. Denn die Überschätzung eines einzelnen Ereignismerkmals ist weder ein Schema noch ein Frame. So besteht z. B. ein „Opfer-Schema“ nicht aus unabhängigen, für alle Befragte einzeln aggregierten „Überschätzungen“ von Herkunft, Alter oder Geschlecht der Opfer, sondern aus einer bei jedem Befragten spezifischen Konfiguration dieser Attribute (vgl. Abbildung 2).

Methodisch überzeugender im Hinblick auf Transformationseffekte ist Rhees (1997) Experiment zu strategischem versus thematischem Framing. Die Studie erfasste Rezipientenvorstellungen sowohl vor als auch nach der Stimulusgabe. Damit ließ sich der intervenierende Einfluss vorbandener Kognitionen der Probanden ermitteln. Indem die Probanden Briefe über die betreffende Bürgermeisterwahl an Freunde schrieben, war die Erfassung ihrer Kognitionen recht umfassend. Auch das Experiment von Shah et al. (1996, 2001) zur Wirkung von Value-Framing berücksichtigte den intervenierenden Einfluss bereits bestehender Wertestandards von Probanden.

\subsubsection{Effekte auf Einstellungen, Meinungen und Gefüble von Rezipienten}

Die meisten der bereits besprochenen Studien fokussierten letztlich auf die Veränderung von Einstellungen oder Meinungen der Rezipienten. Die relative Mehrzahl der Studien beleuchtet Urteile über Politiker. Arbeiten des Attribute-Agenda-Setting (z. B. Golan \& Wanta, 2001; McCombs et al., 1997) vergleichen nur die Gewichtung „affektiver Einzelmerkmale“ von Präsidentschaftskandidaten bei Medien und Rezipienten. Brauchbarer ist demgegenüber die Studie von Park \& Kosicki (1995) zur Iran-Contra-Affäre. Sie ermittelten den Einfluss von „valence“ und „position frames“ auf die Urteile über Reagan. Die Medien-Frames spielten dafür zwar eine Rolle, durchschlagender waren aber Parteipräferenz und Präsidenten-Images.

Komplexere Wirkungszusammenhänge untersuchte Iyengar (1991). Seine Experimente zu episodischem und thematischem Framing gaben Hinweise auf Spill-Over-Effekte von themenbezogenen Kausalattributionen auf weiter gehende Urteile (Iyengar, 1991: 82ff.). So wurde der US-Präsident z. B. in Bezug auf Armut negativer beurteilt, wenn Armut als gesellschaftliches Problem gesehen wurde; der beste Prädiktor für Ur- 
teile über Reagan war aber die Parteipräferenz. Auch Iyengars (1991: 69ff., 95ff.) Experiment zur Iran-Contra-Affäre belegte Spill-Over-Effekte. Urteile über Reagans Außenpolitik oder über seine Vorgehensweise gegen Terrorismus fielen negativer aus, wenn dem US-Präsidenten die Verantwortung für den Waffenexport in den Iran zugeschrieben wurde. Die Befunde legen ebenso ein Zwei-Stufen-Modell nahe wie das Experiment von Shah et al. (1996, 2001). Wie erwähnt, schienen der ethische bzw. der materialistische Medien-Frame im ersten Schritt jeweils andere Werte-Standards bei den Probanden zu aktivieren. Im zweiten Schritt beeinflussten diese dann auch deren Präferenzen für einen Kandidaten.

Einstellungen zu Personen und Meinungen zu Sachverhalten waren die abhängigen Variablen bei Nelson et al. (1997). Wirkungen auf themengebundene Meinungen - nämlich die Zustimmung zu einer militärischen bzw. diplomatischen Intervention - betrachteten Iyengar \& Simon (1993). In einem weiteren Verständnis kann man auch die Wirkung von Framing auf Politikverdrossenheit als Einstellungs-Effekt begreifen. Cappella \& Jamieson (1997) konnten den Zusammenhang zwischen strategischem Framing und Politikverdrossenheit aber nicht eindeutig nachweisen.

Schließlich scheint Medien-Framing auch Auswirkungen auf die Gefüble der Rezipienten zu haben. In einem zweiten Experiment bei Price et al. (1997) sollten die Probanden ihre Gefühle zu Personen und Institutionen der Universität sowie ihre Ansichten zu Studiengebühren und Universität angeben. Varianzanalysen und vergleichende multivariate Analysen gaben Hinweise darauf, dass diese Urteile und Affekte tatsächlich vom jeweiligen Medien-Frame beeinflusst wurden.

\subsection{Methodische und empirische Bestandsaufnabme}

Aus einer synoptischen Betrachtung der von uns ausgewählten empirischen Untersuchungen zu Framing-Effekten ergibt sich folgende methodische und operationale Bestandsaufnahme:

- Kombinationen aus Inbaltsanalyse und Befragung argumentieren oft auf Aggregatdatenniveau und erliegen mitunter einem ökologischen Fehlschluss (z. B. Golan \& Wanta, 2001). Datensätze werden z. T. nicht miteinander verknüpft (z. B. Park \& Kosicki, 1995). Cross-lagged-Panel-Designs oder Zeitreihenanalysen, die synthetische Kausalnachweise (Scheufele, 1999b: 29f.) erlauben, fehlen völlig.

- Experimente nutzen oft Originalbeiträge (u. a. Cappella \& Jamieson, 1997; Price et al., 1997), die teilweise aber nicht nur im Stimulus variieren (z. B. Iyengar, 1991). Ungeklärt ist die Frage der Wirkung durch kumulativ-konsonantes Medien-Framing, mitunter wird der Stimulus aber sequenziell induziert (Rhee, 1997; Cappella \& Jamieson, 1997).

- Erfassung von Rezipienten-Schemata: Relativ häufig werden Rezipientenvorstellungen offen abgefragt und Antworten inhalts- bzw. faktorenanalytisch ausgewertet (z. B. Price et al., 1997; Rhee, 1997; Shah et al., 1996; Valkenburg et al., 1999). Solche Erfassungsmodi gehen weit über Recall oder Recognition von Informationen hinaus und erlauben, Vorstellungswelten bzw. Frames von Rezipienten oder wenigstens Teile davon zu erfassen. Feldstudien greifen meist auf nationale Umfragen zurück, die einzelne Kandidatenmerkmale abfragen, denen die Kategorien der Inhaltsanalysen folgen (z. B. McCombs et al., 1997).

- Pretest: Die Ermittlung von Rezipienten-Schemata in Pretests z. B. Rhee, 1997; Nelson et al., 1997) aktiviert diese automatisch. Durch solches „Priming“ sind sie zugänglich und können die eigentlichen Framing-Effekte überlagern. Ein Solomon- 
Vier-Gruppen-Plan (vgl. Campbell \& Stanley, 1963: 24f.) kann diese Überlagerung zumindest kontrollieren.

Aus einer empirischen Perspektive lassen sich die Befunde der besprochenen Wirkungsstudien zu folgenden Feststellungen bündeln:

- Intervenierende Variablen: Während für Agenda-Setting zentrale intervenierende Variablen feststehen (Brosius, 1994: 271f.), lassen sie sich für Framing nicht abschließend benennen. Bei Rezipienten scheinen folgende Faktoren eine Rolle zu spielen: Politische Einstellungen, Expertise und Aktivität (Iyengar, 1991; Park \& Kosicki, 1995; Nelson et al., 1997), themenbezogene Meinungen, Schemata, Wertestandards (z. B. Nelson et al., 1997; Rhee, 1997; Shah et al., 1996) und Soziodemographie (u. a. Price et al., 1997). Für den Stimulus gibt es Hinweise auf deutlichere Effekte beim Printformat (u. a. Rhee, 1997; Cappella \& Jamieson, 1997) und unterschiedliche Effekte je nach Thema (Iyengar, 1991).

- Zwei-Stufen-Modell: Viele Studien (z. B. Brosius \& Eps, 1995; Früh, 1991b; Rhee, 1997; Valkenburg et al., 1999) belegen ein Zusammenspiel von Medien-Frame und Rezipienten-Schemata. Die Befunde mehrerer Arbeiten (z. B. Iyengar, 1991; Park \& Kosicki, 1995; Nelson et al., 1997; Price et al., 1997; Shah et al., 1996) legen zudem ein Zwei-Stufen-Modell nahe: Medien-Frames beeinflussen im ersten Schritt die Kognitionen (Aktivierung, Etablierung, Veränderung von Schemata) und im zweiten Schritt die Urteile, Einstellungen, Meinungen, Gefühle und Entscheidungen von Rezipienten.

\section{Offene Fragen und Anknüpfungspunkte}

Eine kritische Auseinandersetzung mit bestehenden Studien ist stets durch konstruktive methodische Vorschläge zu ergänzen.

\section{Experimente und Feldstudien}

Experimentelle Studien messen die Wirkung des Medien-Frames meist direkt nach der Stimulusinduzierung. Dies geht an tatsächlichen Urteils- bzw. Entscheidungssituationen vorbei, wie sie der Framing-Ansatz beschreibt. Extern valider wäre, die jeweilige abhängige Variable z. B. erst eine Woche nach Stimulusgabe zu erfassen. Oft wird der Stimulus auch nur einmalig dargeboten, womit sich kurzfristige Aktivierungs-Effekte nachweisen lassen. Etablierungs-, Transformations- und Einstellungs-Effekte, die sich mittel- bis langfristig entfalten, lassen sich dagegen selbst durch sequenzielle Stimulusdarbietung höchstens näherungsweise belegen. Zusammenfassend sollten sich kommunikationswissenschaftliche Framing-Effekt-Studien von ihrer (impliziten) Orientierung an den klassischen Framing-Studien der "prospect theory“ (u. a. Kahneman \& Tversky, 1979, 1984; Tversky \& Kahneman, 1990) verabschieden und in ihren Untersuchungsdesigns stärker die Charakteristika der Medienberichterstattung und Medienrezeption berücksichtigen.

Für weitere Fragen können wir Lösungen nur skizzieren: Der Abstraktionsgrad der Schemata von Rezipienten ist empirisch zu bestimmen - etwa so, wie wir es in Abschnitt 2 vorgeschlagen haben. Die in bisherigen Experimenten verwendeten Frame-Stimuli sind entweder eher Nachrichtenfaktoren (z. B. Price et al., 1997; Rhee, 1997; Valkenburg et al., 1999) oder theoretisch festgelegt. Hier wären inhaltsanalytische Voranalysen sinnvoll, um Stimuli zu generieren, die tatsächlichen Medien-Frames entsprechen. Experimente mit formalen Frames kontrollierten selten eine Konfundierung des ab- 
strakten Frames mit inhaltlichen Aspekten - etwa der Art der Opfer (Iyengar, 1991). Hier wären zwei- oder mehrfaktorielle Designs angeraten, die z. B. beim Thema „Terrorismus" auf dem ersten Faktor formales Framing (z. B. episodisch vs. thematisch) und auf dem zweiten Faktor inhaltliches Framing (z. B. „Religiöser Fanatismus“ vs. „Nahost-Politik“) variieren. Transformations-Effekte lassen sich wiederum durch Messwiederholungen nachweisen.

Für Feldstudien im Längsschnittsdesign sind zwei Fälle zu unterscheiden: (1) Umfrageinstitute erheben kontinuierlich Einstellungen und Meinungen, deren Veränderung sich mit Veränderungen im Medien-Framing verknüpfen lassen. Mit Cross-lagged-Panel-Designs oder Zeitreihenanalysen würde man Kausalzusammenhänge synthetisch prüfen (Scheufele, 1999b: 172ff.). Allerdings wären auch Mediennutzungsdaten heranzuziehen, um das Aggregatdatenproblem zu mindern. (2) Für Framing-Effekte auf kognitive Vorstellungen unterliegen Feldstudien einigen Beschränkungen: Umfrageinstitute stellen routinemäßig die Agenda-Setting-Frage. Mit einer vergleichbar simplen Frage lassen sich themengebundene Schemata aber kaum ermitteln. Hier wären offene Fragen wie beim „thought listing“ (Price et al., 1997) sinnvoll; dazu gleich mehr. Ein solcher Erhebungsmodus wäre nur für wenige Zeitpunkte überhaupt praktikabel. Eine Alternative wäre, die Schemata der Rezipienten dann zu ermitteln, wenn sich signifikante Veränderungen im Medien-Framing zeigen. Solche Veränderungen lassen sich jedoch nur ex post feststellen. Damit wären auch die Messzeitpunkte der Befragung retrospektiv festzulegen, was nicht möglich ist.

Die routinemäßige Erfassung von Schemata ist schwierig. Ein Standard-Instrument wäre aber für regelmäßig wiederkehrende Themen (z. B. Wahlen oder Kriege) denkbar. Um Standard-Fragen zur Erfassung von Rezipienten-Schemata z. B. bei Wahlen zu entwickeln, wäre eine Meta-Analyse vieler, erst einmal zu projektierender Studien nötig, die mit Gedankenprotokollen (vgl. Price et al., 1997) arbeiten. Aus der Gesamtschau dieser Gedankenprotokolle könnte man einen Katalog von Schema-Typen extrahieren, die Rezipienten üblicherweise auf Wahlkämpfe, wahlrelevante Themen und Kandidaten anwenden. Für Umfragen könnte man daraus Item-Batterien von Indikatoren für diese Schemata umsetzen (vgl. Scheufele, 2003: 225).

\section{Tendenzen, Frame-Elemente und Strukturmerkmale der Medienberichterstattung}

Näher zu beleuchten ist das Zusammenspiel aus Tendenz und Rahmung. Vermutlich dürften sich für die von Park \& Kosicki (1995) untersuchten „valence frames“ stärkere Framing-Effekte als für „position frames“ ergeben. Zudem kann die kumulative und konsonante mediale Rahmung eines Themas, die mit einem klaren Tenor einhergeht, dazu führen, dass Rezipienten überhaupt erst eine kognitiv-affektive Verbindung zwischen dem Problem und einer Position dazu herausbilden.

Wirkungsstudien operationalisieren Medien-Frames meist eindimensional, womit sie wiederum dem Experimentaldesign der erwähnten „prospect theory“ folgen. Völlig ignoriert werden damit allerdings zentrale Überlegungen z. B. der öffentlichkeits- und bewegungstheoretischen Framing-Perspektive (dazu Scheufele, 2003: 84ff.). Studien hierzu (u. a. Gamson, 1992; Gamson \& Modigliani, 1989; Gerhards et al., 1998; Neidhardt \& Rucht, 1993; Snow \& Benford, 1988; Weßler, 1999) betonen einhellig, dass ein (kollektiver) Frame diverse Elemente umfasst - z. B. Problemdefinition, Kausalzusammenhänge, Geltungsansprüche oder Werte. Nur wenn diese ein konsistentes Bild vermitteln, entfaltet der Frame normative Bindungskraft. Möglicherweise sind aber auch einzelne Elemente in bestimmten thematischen Zusammenhängen wirksamer als ande- 
re. Dies müsste für unterschiedliche Themen geprüft werden. So dominieren z. B. bei Unglücken Fragen nach Ursachen und Verantwortlichen (Snow \& Benford, 1988: 200ff.; Gerhards \& Neidhart, 1990: 42); hier dürfte v. a. kausales Framing durchschlagen. Politische Skandale zeichnet aus, dass Verhalten und Ansprüche von Politikern auseinander klaffen (Neckel, 1990: 3); hier dürfte eine Rahmung über Geltungsansprüche wirksamer sein, schon weil damit an Werte appelliert wird. Darüber hinaus wäre zu prüfen, ob sich Framing-Effekte nur bei kontroversen Themen einstellen. Bei Routinethemen dürften - mit Befunden zu chronischer Zugänglichkeit von Schemata (Higgins, 1989) - die bestehenden Vorstellungen von Rezipienten deutlich intervenieren.

Auch Strukturmerkmale der Berichterstattung sind stärker einzubinden: So zeigen z. B. psychologische Befunde, dass Rezipienten bei thematisch einheitlichen Texten häufiger Inferenzen ziehen (Schnotz, 1985: 291ff.). Übertragen auf Framing könnte also der Grad an Frame-Konsistenz eines Beitrags die Effektstärke beeinflussen; vermutlich lässt sich auch ein Schwellenwert ausmachen. ${ }^{12}$ Spezifische Effekte sind für das visuelle Format zu erwarten (Scheufele, 1999a: 100f.; 2001a: 150f.). Dies legen Studien nahe, wonach Rezipienten bei unterschiedlichen Bildern andere Urteile über Politiker fällen (z. B. Spignesi \& Shor, 1981; Baggaley, 1980). Berührungspunkte bestehen auch mit Studien zum Verhältnis von Text und Bild (Brosius, 1998): Ein verbaler und ein visueller Frame können additiv wirken oder auseinander klaffen (Text-Bild-Schere). Auch das zentrale Charakteristikum des Fernsehens, nämlich die sequenzielle Abfolge von Bildern und Filmsequenzen, legt Framing- bzw. Priming-Effekte nahe. Dabei wäre zu prüfen, ob Frames nur „proaktiv“ oder auch „retrospektiv rahmen“. Effekte visueller Frames wurden bislang so gut wie nicht untersucht, obwohl viele Arbeiten (z. B. Entman, 1991; Gamson \& Modigliani 1989; Pan \& Kosicki, 1993) die Bedeutung visueller Elemente betonen. Die wenigen Ausnahmen sind einzelfallbezogen (z. B. Gamson \& Modigliani, 1989), untersuchen visuelle Darstellungen nur in Printmedien, zählen lediglich Karikaturen und Grafiken aus (z. B. Entman, 1991) oder sind bei hermeneutischer Ausrichtung (z. B. Kress \& van Leeuwen, 1998) nicht aussagekräftig (Scheufele, 1999a, 2001a).

\section{Integration verschiedener Forschungsstränge des Framing-Ansatzes}

Gerade öffentlichkeitstheoretische Framing-Studien (z. B. Gerhards et al., 1998; vgl. Abschnitt 1) setzen die Frage medialer Breitenwirkung nicht hinreichend um. Dies liegt auch teilweise daran, dass sie Medien nur als Diskurs-Arena betrachten, womit sie ihnen Wirkungspotenzial absprechen. Umgekehrt betrachtet der wirkungszentrierte Framing-Ansatz die Medien zwar als zentrale Einflussgröße. Allerdings dominieren bislang mikropsychologische Wirkungskonzepte. Dabei werden weder Elemente und Ebenen medialer Frames noch gesellschaftliche oder politische Implikationen von Medien-Framing berücksichtigt. Dabei wurden gesamtgesellschaftliche Medienwirkungen unter dem Etikett „Agenda-Setting als sozialer Prozess“ (Eichhorn, 1996: 111ff.) bereits problematisiert. Auf ähnliche Weise wäre die psychologisch ausgerichtete Wirkungsperspektive des Framing-Ansatzes stärker für soziologische und diskurstheoretische Fragen zu öffnen.

12 Dabei ist allerdings auch der intervenierende Einfluss kognitiver Fähigkeiten (u. a. Wirth, 1997: 56ff.; Park \& Kosicki, 1995) zu berücksichtigen. 


\section{Literaturverzeichnis}

Alba, J. \& Hasher. L. (1983): Is memory schematic? In: Psychological Bulletin 93, S. 203-231.

Axelrod, R. (1973): Schema theory: An information processing model of perception and cognition. In: American Political Review 67, S. 1248-1266.

Baggaley, J. (1980): Psychology of the TV image. Westmead.

Benton, M. \& Frazier, P. J. (1976): The agenda-setting of the mass media at three levels of „information-holding“. In: Communication Research 3, S. 261-274.

Bohner, G. / Erb, H.-P. \& Crow, K. (1995): Priming und Persuasion: Einflüsse der Aktivierung verschiedener Persönlichkeitsdimensionen auf Prozesse der Einstellungsänderung und auf die Beurteilung des Kommunikators. In: Zeitschrift für Sozialpsychologie 26, S. 263271.

Brosius, H.-B. (1991): Schema-Theorie - ein brauchbarer Ansatz in der Wirkungsforschung? In: Publizistik 36, S. 285-297.

Brosius, H.-B. (1994): Agenda-Setting nach einem Vierteljahrhundert Forschung: Methodischer und theoretischer Stillstand? In: Publizistik 39, S. 269-288.

Brosius, H.-B. (1995): Alltagsrationalität in der Nachrichtenrezeption. Ein Modell der Wahrnehmung und Verarbeitung von Nachrichteninhalten. Opladen.

Brosius, H.-B. (1998): Visualisierung von Fernsehnachrichten. Text-Bild-Beziehungen und ihre Bedeutung für die Informationsleistung. In: Kamps, K. \& Meckel, M. (Hrsg.): Fernsehnachrichten. Prozesse, Strukturen, Befunde. Opladen. S. 213-224.

Brosius, H.-B. \& Eps, P. (1993): Verändern Schlüsselereignisse journalistische Selektionskriterien? Framing am Beispiel der Berichterstattung über Anschläge gegen Ausländer und Asylanten. In: Rundfunk \& Fernsehen 41, S. 512-530.

Brosius, H.-B. \& Eps, P. (1995): Framing auch beim Rezipienten? Der Einfluß der Berichterstattung über fremdenfeindliche Anschläge auf die Vorstellungen der Rezipienten. In: Medienpsychologie 7, S. 169-183.

Campbell, D. T. \& Stanley, J. C. (1963): Experimental and quasi-experimental designs for research. Chiacgo.

Cappella, J. N. \& Jamieson, K. H. (1996): News frames, political cynicism, and media cynicism. In: Jamieson, K. H. (Hrsg.): The Annals of the American Academy of Political and Social Science 546, S. 71-84.

Cappella, J. N. \& Jamieson, K. H. (1997): Spiral of cynicism. The press and the public good. New York, Oxford.

Clark, A. (1989): Microcognition: Philosophy, cognitive science, and parallel distributed processing. Cambridge (Mass).

Cohen, B. C. (1963): The Press and foreign policy. Princeton.

Collins, A. M. \& Loftus, E. F. (1975): A spreading-activation theory of semantic processing. In: Psychological Review 82, S. 407-428.

Daschmann, G. (2001): Der Einfluß von Fallbeispielen auf Leserurteile. Experimentelle Untersuchungen zur Medienwirkung (= Wissenschaftsforum: Publizistik - Kommunikationswissenschaft - Medien; Bd. 8). Konstanz.

Dijk, T. A. van (1988): News as discourse. Hillsdale (NJ).

Dijk, T. A. van \& Kintsch, W. (1983): Strategies of discourse comprehension. New York.

Dörffner, G. (1991): Konnektionismus. Stuttgart.

Eichhorn, W. (1996): Agenda-Setting-Prozesse. Eine theoretische Analyse individueller und gesellschaftlicher Themenstrukturierung. München.

Entman, R. M. (1991): Framing U.S. coverage of international news: Contrasts in narratives of the KAL and Iran air incidents. In: Journal of Communication 41, S. 6-27.

Entman, R. M. (1993): Framing: Towards clarification of a fractured paradigm. In: Journal of Communication 43 , S. 51-58.

Fishbein, M. \& Ajzen, L. (1975): Belief, attitude, intention and behavior. An introduction to theory and research. Reading (MA).

Fiske, S. T. (1982): Schema-triggered affect: Applications to social perception. In: Clark, M. S. \& 
Fiske, S. T. (Hrsg.): Affect and cognition: The 17th Annual Carnegie Symposium on Cognition. Hillsdale (NJ). S. 55-78.

Fiske, S. T. \& Taylor, S. E. (1991): Social Cognition. 2. Auflage. New York u.a.

Früh, W. (1991a): Medienwirkungen: Das dynamisch-transaktionale Modell. Theorie und empirische Forschung. Opladen.

Früh, W. (1991b): Der aktive Rezipient - neu besehen. Zur Konstruktion faktischer Information bei der Zeitungslektüre. In: Früh, W.: Medienwirkungen: Das dynamisch-transaktionale Modell. Theorie und empirische Forschung. Opladen. S. 237-258 (zuerst veröffentlicht in: Publizistik 28, 1983, S. 327-342).

Früh, W. \& Schönbach, K. (1982): Der dynamisch-transaktionale Ansatz. Ein neues Paradigma der Medienwirkungen. In: Publizistik 27, S. 74-88.

Gamson, W. A. (1992): Talking politics. New York.

Gamson, W. A. \& Modigliani, A. (1989): Media discourse and public opinion on nuclear power: A constructionist approach. In: American Journal of Sociology 95, S. 1-37.

Gerbner, G./Morgan, M. \& Signorelli, N. (1999): Profiling television violence. In: Nordenstreng, K. (Hrsg.): International media monitoring. Cresskill (NJ). S. 335-365.

Gerhards, J. \& Neidhardt, F. (1990): Strukturen und Funktion moderner Öffentlichkeit. Fragestellungen und Ansätze. Wissenschaftszentrum für Sozialforschung Berlin. Unveröffentlichtes Discussionpaper FS III 90-101. Berlin.

Gerhards, J./Neidhardt, F. \& Rucht, D. (1998): Zwischen Palaver und Diskurs. Strukturen öffentlicher Meinungsbildung am Beispiel der deutschen Diskussion zur Abtreibung. Opladen, Wiesbaden.

Ghanem, S. (1997): Filling the tapestry: The second level of agenda-setting. In: McCombs, M./Shaw, D. L. \& Weaver, D. (Hrsg.): Communication and democracy. Exploring the intellectual frontiers in agenda-setting-theory. Mahawah (NJ), London. S. 3-14.

Gitlin, T. (1980): The whole world is watching: Mass media in the making \& unmaking of the new left. Berkeley.

Goffman, E. (1993): Rahmen-Analyse. Ein Versuch über die Organisation von Alltagserfahrungen. 3. Auflage. Frankfurt am Main [1974].

Golan, G. \& Wanta, W. (2001): Second-level agenda-setting in the New Hampshire primary: A comparison of coverage in three newspapers and public perceptions of candidates. In: Journalism and Mass Communication Quarterly 78, S. 247-259.

Hastie, R. (1981): Schematic principles in human memory. In: Higgins, E. T./Herman, C. P. \& Zanna, M. P. (Hrsg.): Social cognition. The Ontario Symposium. Vol. 1. Hillsdale (NJ). S. 39-89.

Higgins, E. T. (1989): Knowledge accessibility and activation: Subjectivity and suffering from unconscious sources. In: Uleman, J. S. \& Bargh, J.A. (Hrsg.): Unintended thought: The limits of awareness, intention and control. New York. S. 75-123.

Higgins, E. T./Herman, C. P. \& Zanna, M. P. (Hrsg.) (1981): Social cognition. The Ontario Symposium. Vol. 1. Hillsdale (NJ).

Higgins, E. T./Bargh, J. A. \& Lombardi, W. J. (1985): Nature of priming effects on categorization. In: Journal of Experimental Psychology: Learning, Memory, and Cognition 11, S. 59-69.

Higgins, E. T. \& Brendl, C. M. (1995): Accessibility and applicability: Some „activation rules“ influencing judgment. In: Journal of Experimental Psychology 31, S. 218-243.

Higgins, E. T. \& Chaires, W. M. (1980): Accessibility of interrelational constructs: Implications for stimulus encoding and creativity. In: Journal of Personality and Social Psychology 16, S. 348-361.

Higgins, E. T./Rholes, W. S. \& Jones, C. R. (1977): Category accessibility and impression formation. In: Journal of Personality and Social Psychology 13, S. 141-154.

Hovland, C. I./Janis, I. L. \& Kelley, H. H. (1953): Communication and persuasion. New Haven. Iyengar, S. (1991): Is anyone responsible? How television frames political issues. Chicago, London. Iyengar, S. \& Kinder, D. R. (1987): News that matters: Television and American opinion. Chicago. Iyengar, S. \& Simon, A. (1993): News coverage of the Gulf crisis and public opinion. A study of agenda setting, priming and framing. In: Communication Research 20, S. 365-383. 
Jasperson, A. E./Shah, D. H./Watts, M./Faber, R. J. \& Fan, D. P. (1998): Framing effects and the importance of the federal budget deficit. In: Political Communication 15, S. 205-224.

Johnson-Laird, P. N. (1983): Mental models. Towards a cognitive science of language, inference, and consciousness. Cambridge.

Johnson-Laird, P. N. (1989): Mental models. In: Posner, M. I. (Hrsg.): Foundations of cognitive science. Cambridge (MA). S. 469-499.

Kahneman, D. \& Tversky, A. (1979): Prospect theory: An analysis of decision under risk. In: Econometrica 47, S. 263-291.

Kahneman, D. \& Tversky, A. (1984): Choices, values, and frames. In: American Psychologist, 39, S. $341-350$.

Kelley, H. H. (1972): Causal schemata and the attribution process. In: Jones, E. E./Kanouse, D. E./Kelley, H. H./Nisbett, R. E. \& Valins, S. \& Weiner, B. (Hrsg.): Attribution: Perceiving the causes of behavior. Morristown (NJ). S. 151-174.

Kepplinger, H. M./Brosius, H.-B. \& Staab, J. F. (1991): Opinion formation in mediated conflicts and crisis: A theory of cognitive-affective media effects. In: International Journal of Public Opinion Reserach 3, S. 132-156.

Kepplinger, H. M./Gotto, K./Brosius, H.-B. \& Haak, D. (1989): Der Einfluß der Fernsehnachrichten auf die politische Meinungsbildung. Freiburg, München.

Kinder, D. R. \& Sanders, L. M. (1990): Mimicking political debate with survey questions: The case of white opinion on affirmative action for blacks. In: Social Cognition 8, S. 73-103.

Kleiber, G. (1998): Prototypensemantik. Eine Einführung. Tübingen.

Kliment, T. (1998): Durch Dramatisierung zum Protest? Theoretische Grundlegung und empirischer Ertrag des Framing-Konzepts. In: Hellmann, K.-U. \& Koopmans, R. (Hrsg.): Paradigmen der Bewegungsforschung. Entstehung und Entwicklung von neuen sozialen Bewegungen und Rechtsextremismus. Opladen. S. 69-89.

Kress, G. \& van Leeuwen, T. (1998): Front pages: (The critical) analysis of newspaper layout. In: Bell, A. \& Garrett, P. (Hrsg.): Approaches to media discourse. Oxford, Malden. S. 186-219.

Lazarsfeld, P. F. \& Barton, B. A. (1951): Qualitative measurement in the social sciences. Classification, typologies, and indices. In: Lerner, D. (Hrsg.): The policy sciences. Stanford. S. 155-192.

Levin, I. P./Schneider, S. L. \& Gaeth, G. J. (1998): All frames are not created equal: A typology and critical analysis of framing effects. In: Organizational Behavior and Human Decision Processes 76, S. 149-188.

Lindsey, P. M. \& Norman, D. A. (1977): Human information processing. An introduction to psychology. New York.

Löffelholz, M. (2000) (Hrsg.): Theorien des Journalismus. Ein diskursives Handbuch. Opladen.

Lombardi, W. J./Higgins, E. T. \& Bargh, J. A. (1987): The role of consciousness in priming effects on categorization. Assimilation versus contrast as a function of awareness of the priming task. In: Personality and Social Psychology Bulletin 13, S. 411-429.

Lüders, C. (1991): Deutungsmusteranalyse. Annäherung an ein risikoreiches Konzept. In: Garz, D. \& Kraimer, K. (Hrsg.): Qualitativ-empirische Sozialforschung. Opladen. S. 377-408.

Manheim, J. B. (1986): A model of agenda dynamics. In: McLaughlin, M. L. (Hrsg.): Communication Yearbook Vol. 10. London. S. 499-516.

McClelland, J. L. \& Rumelhart, D. E. (1986) (Hrsg.): Parallel distributed processing. Vol. 2: Psychological and biological models. Cambridge (MA).

McCombs, M. \& Ghanem, S. I. (2001): The convergence of agenda setting and framing. In: Reese, S. D./Gandy, Oscar H. \& Grant, A. E. (Hrsg.): Framing public life. Perspectives on media and our understanding of the social world. Mahwah (NJ), London. S. 67-81.

McCombs, M. E. \& Shaw, D. L. (1972): The agenda-setting function of mass media. In: Public Opinion Quarterly 36, S. 176-187.

McCombs, M./Llamas, J. P./Lopez-Escobar, E. \& Rey, F. (1997): Candidate images in Spanish elections: Second-level agenda-setting effects. In: Journalims \& Mass Communication Quarterly 74, S. 703-717.

McGuire, W. J. (1985): Attitudes and attitude change. In: Lindzey, G. \& Aronson, E. (Hrsg.): Handbook of Social Psychology. Vol. 2. New York. S. 233-346. 
Neckel, S. (1990): Die Wirkungen politischer Skandale. In: Aus Politik und Zeitgeschichte (Beilage zur Wochenzeitung „Das Parlament“) Heft B7/90 vom 9. Februar 1990, S. 3-10.

Neidhardt, F. \& Rucht, D. (1993): Auf dem Weg in die „Bewegungsgesellschaft“? In: Soziale Welt 44 , S. 305-326.

Nelson, T. E./Oxley, Z. M. \& Clawson, R. A. (1997): Toward a psychology of framing effects. In: Political Behavior 19, S. 221-246.

Neuman, W. R./Just, M. R. \& Crigler, A. N. (1992): Common knowledge. News and the construction of political meaning. Chicago, London.

Noelle-Neumann, E. (1973): Kumulation, Konsonanz und Öffentlichkeitseffekt. Ein neuer Ansatz zur Analyse der Wirkung der Massenmedien. In: Publizistik 18, S. 26-55.

Noelle-Neumann, E. \& Mathes, R. (1987): The „event as event“ and the „event as news“: The significance of "consonance “ for media effects research. In: European Journal of Communication 2, S. 391-414.

Pan, Z. \& Kosicki, G. M. (1993): Framing analysis: An approach to news discourse. In: Political Communication 10, S. 55-75.

Pan, Z. \& Kosicki, G. M (1997): Priming and media impact on the evaluations of the president's performance. In: Communication Research 24, S. 3-30.

Park, E. \& Kosicki, G. M. (1995): Presidential support during the Iran-contra-affair: People's reasoning process and media influence. In: Communication Research 22, S. 207-236.

Patterson, T. (1993): Out of Order. New York.

Peeters, G. (1991): Evaluative inference in social cognition: The role of direct versus indirect evaluation and positive-negative asymmetry. In: European Journal of Social Psychology 21, S. 131-146.

Peter, J. (2002): Medien-Priming - Grundlagen, Befunde und Forschungstendenzen. In: Publizistik 47, S. 21-44.

Price, V. \& Tewksbury, D. (1997): News values and public opinion: A theoretical account of media priming and framing. In: Barnett, G. \& Boster, F. J. (Hrsg.): Progress in the communication sciences. Greenwich (CT). S. 173-212.

Price, V./Tewksbury, D. \& Powers, E. (1997): Switching trains of thought: The impact of news frames on reader's cognitive responses. In: Communication Research 24, S. 481-506.

Ratcliff, R. A. \& McKoon, G. (1978): Priming an item recognition. Evidence for the propositional structure in sentences. In: Journal of Verbal Learning and Verbal Behavior 17, S. 403-417.

Reese, S. D. (2001): Prologue - Framing public life: A bridging model for media research. In: Reese, S. D./Gandy, Oscar H. \& Grant, A. E. (Hrsg.): Framing public life. Perspectives on media and our understanding of the social world. Mahwah (NJ), London. S. 7-31.

Rhee, J. W. (1997): Strategy and issue frames in election campaign coverage: A social cognitive account of framing effects. In: Journal of Communication 47, S. 26-48.

Rössler, P. (1997): Agenda-Setting. Theoretische Annahmen und empirische Evidenzen einer Medienwirkungshypothese (Studien zur Kommunikationswissenschaft, Bd. 27). Opladen.

Rogers E. M. \& Dearing, J. W. (1988): Agenda-setting research: Where has it been, where is it going? In: Anderson, J. H. (Hrsg.): Communication Yearbook. Vol. 11. Newbury Park, Beverly Hills, London, New Delhi. S. 555-594.

Rosch E. H./Mervis, C. B./Johnson, D. \& Boyes-Braem, P. (1976): Basic objects in natural categories. In: Cognitive Psychology 8, S. 573-605.

Rosenberg, M. J. (1956): Cognitive structure and attitudinal affect. In: Journal of Abnormal Social Psychology 53, S. 367-372.

Rumelhart, D. E. \& Norman, D. A. (1978): Accretion, tuning, and restructuring: Three models of learning. In: Cotton, J. W. \& Klatzky, R. L. (Hrsg.): Semantic factors in cognition. Hillsdale (NJ). S. 37-53.

Scheufele, B. (1999a): (Visual) Media Framing und Politik. Zur Brauchbarkeit des Framing-Ansatzes im Kontext (visuell) vermittelter politischer Kommunikation und Meinungsbildung. In: Hofmann, W. (Hrsg.): Die Sichtbarkeit der Macht. Theoretische und empirische Untersuchungen zur visuellen Politik. Baden-Baden, S. 91-107. 
Scheufele, B. (1999b): Zeitreihenanalysen in der Kommunikationsforschung. Eine praxisorientierte Einführung in die univariate und multivariate Zeitreihenanalyse mit SPSS for Windows. Stuttgart.

Scheufele, B. (2000): „Scattered“ or related - clarifying the framing concept by integrating related approaches? In: Brosius, H.-B. (Hrsg.): Kommunikation über Grenzen und Kulturen. - Konstanz. S. 381-396.

Scheufele, B. (2001a): Visuelles Medien-Framing und Framing-Effekte. Zur Analyse visueller Kommunikation aus der Framing-Perspektive. In: Knieper, T. \& Müller, M. G. (Hrsg.): Kommunikation visuell - Das Bild als Forschungsgegenstand - Grundlagen und Perspektiven. Köln. S. 144-158.

Scheufele, B. (2001b): Notwendigkeit, Nutzen und Aufwand von Mehrfach- und Sondercodierungen. In: Wirth, W. \& Lauf, E. (Hrsg.): Inhaltsanalyse. Perspektiven, Probleme, Potentiale. Köln: Herbert von Halem Verlag. S. 82-97.

Scheufele, B. (2003): Frames - Framing - Framing-Efekte. Theoretische und methodische Grundlegung sowie empirische Befunde zur Nachrichtenproduktion. Wiesbaden.

Scheufele, D. (1999): Framing as a theory of media effects. In: Journal of Communication 49, S. $103-122$.

Schnotz, W. (1985): Selectivity in drawing inferences. In: Rickheit, G. \& Strohner, H. (Hrsg.): Inferences in text processing. Amsterdam, New York, Oxford. S. 287- 326.

Schnotz, W. (1994): Aufbau von Wissensstrukturen. Untersuchungen zur Kohärenzbildung bei Wissenserwerb mit Texten (Fortschritte der psychologischen Forschung; Bd. 20). Weinheim.

Schütz, A. (1971): Gesammelte Aufsätze. Band 1: Das Problem der Wirklichkeit. Den Haag.

Schütz, A. (1993): Der sinnhafte Aufbau der sozialen Welt. Eine Einleitung in die verstehende Soziologie. 6. Auflage. Frankfurt am Main [1932].

Shah, D. V./Domke, D. \& Wackman, D. B. (1996): „To thine own self be true“. Values, framing, and voter decision-making strategies. In: Communication Research 23, S. 509-560.

Shah, D. V./Domke, D. \& Wackman, D. B. (2001): The effects of value-framing on political judgment and reasoning. In: Reese, S. D./Gandy, Oscar H. \& Grant, A. E. (Hrsg.): Framing public life. Perspectives on media and our understanding of the social world. Mahwah (NJ), London. S. $227-243$.

Signorelli, N. \& Morgan, M. (1990) (Hrsg.): Cultivation analysis: New directions in media effects research. Newbury Park.

Snow, D. A. \& Benford, R. D. (1988): Ideology, frame resonance, and participant mobilization. In: Klandermans, B./Kriesi, H./Tarrow, S. (Hrsg.): International social movement research, Vol. 1: From structure to action: Comparing social movement research across cultures. Greenwich (CT). S. 197-217.

Snow, D. A. \& Benford, R. D. (1992): Master frames and cycles of protest. In: Morris, A. D. \& Mueller, C. M. (Hrsg.): Frontiers in social movement theory. New Haven (CT). S. 133-155.

Spignesi, A. \& Shor R. E. (1981): The judgement of emotion from facial expressions, contexts, and their combination. In: The Journal of General Psychology 104, S. 41-58.

Srull, T. K. \& Wyer, R. S. (1979): The role of category accessibility in the interpretation of information about persons: Some determinants and implications. In: Journal of Personality and Social Psychology 37, S. 1660-1672.

Staab, J. F. (1990): Nachrichtenwert-Theorie. Formale Struktur und empirischer Gehalt (AlberReihe: Kommunikation; Bd. 17). Freiburg, München.

Staab J. F. (1992): Ausstrahlungseffekte von Beiträgen in Fernsehnachrichten. Zur Ursachenattribution bei der Rezeption politischer Medieninhalte. In: Rundfunk \& Fernsehen 40, S. 544-556.

Stoffer, T. H. (1990): Perspektiven konnektionistischer Modelle. Das neuronale Netzwerk als Metapher im Rahmen der kognitionspsychologischen Modellbildung. In: Meinecke, C. \& Kehrer, L. (Hrsg.): Bielefelder Beiträge zur Kognitionspsychologie. Göttingen. S. 275-304.

Tankard, J. W. (2001): The empirical approach to the study of media framing. In: Reese, S. D./Gandy, Oscar H. \& Grant, A. E. (Hrsg.): Framing public life. Perspectives on media and our understanding of the social world. Mahwah (NJ), London. S. 95-106.

Takeshita, T. (1997): Exploring the media's roles in defining reality: From issue-agenda-setting to 
attribute-agenda-setting. In: McCombs, M./Shaw, D. L. \& Weaver, D. (Hrsg.): Communication and democracy. Exploring the intellectual frontiers in agenda-setting-theory. Mahawah (NJ), London. S. 15-27.

Tesser, A. (1978): Self-generated attitude change. In: Berkowitz, L. (Hrsg.): Advances in experimental social psychology. Vol 11. New York. S. 289-233.

Tuchman, G. (1972): Making news by doing work: Routinizing the unexpected. In: American Journal of Sociology 79, S. 110-131.

Tuchman, G. (1978): Making news. A study in the construction of reality. New York.

Tversky, A. \& Kahneman, D. (1990): Rational choice and the framing of decisions. In: Cook, K. S. \& Levi, M. (Hrsg.): The limits of rationality. Chicago. S. 50-89.

Valkenburg, P. M./Semetko, H. A. \& Vreese, C. H (1999): The effects of news frames on readers's thoughts and recall. In: Communication Research 26, S. 550-569.

Weber, M. (1988): Die „Objektivität“ sozialwissenschaftlicher und sozialpolitischer Erkenntnis [1904]. In: Weber, M.: Gesammelte Aufsätze zur Wissenschaftslehre. Herausgegeben von Johannes Winkelmann. 7. Auflage. Tübingen. S. 146-214.

Weßler, H. (1999): Öffentlichkeit als Prozess. Deutungsstrukturen und Deutungswandel in der deutschen Drogenberichterstattung. Opladen.

Wicks, R. H. (1992): Schema theory and measurement in mass communication research: Theoretical and methodological issues in news information processing. In: Deetz, S. A. (Hrsg.): Communication Yearbook. Vol. 15. Newbury Park, London, New Delhi. S. 115-145.

Willnat, L. \& Zhu, J.-H. (1996): Newspaper coverage and public opinion in Hong Kong: A timeseries analysis of media priming. In: Political Communication 13, S. 221-246.

Willnat, L. (1997): Agenda setting and priming: Conceptual links and differences. In: McCombs, M./Shaw, D. L. \& Weaver, D. (Hrsg.): Communication and democracy. Exploring the intellectual frontiers in agenda-setting-theory. Mahawah (NJ), London. S. 51-66.

Wirth, W. (1997): Von der Information zum Wissen. Die Rolle der Rezeption für die Entstehung von Wissensunterschieden. Opladen.

Woo, J. (1996): Television news discourse in political transition: Framing the 1987 and 1992 Korean presidential elections. In: Political Communication 13, S. 63-80.

Wyer, R. S. \& Carlston, D. E. (1979): Social cognition, inference, and attribution. Hillsdale (NJ).

Wyer, R. S. \& Srull, T. K. (1981): Category accessibility: Some theoretical and empirical issues concerning the processing of social stimulus information. In: Higgins, E. T./Herman, C. P. \& Zanna, M. P. (Hrsg.): Social cognition. The Ontario Symposium. Vol. 1. Hillsdale (NJ). S. 161-197.

Yagade, A. \& Dozier, D. M (1990): The media agenda-setting effect on concrete versus abstract issues. In: Journalism Quarterly 67, S. 3-10. 